\title{
Stress response regulators identified through genome-wide transcriptome analysis of the $(p)$ ppGpp-dependent response in Rhizobium etli
}

\author{
Maarten Vercruysse, Maarten Fauvart, Ann Jans, Serge Beullens, Kristien Braeken, Lore Cloots, Kristof Engelen, \\ Kathleen Marchal and Jan Michiels
}

\begin{abstract}
Background: The alarmone (p)ppGpp mediates a global reprogramming of gene expression upon nutrient limitation and other stresses to cope with these unfavorable conditions. Synthesis of (p)ppGpp is, in most bacteria, controlled by RelA/SpoT (Rsh) proteins. The role of (p)ppGpp has been characterized primarily in Escherichia coli and several Gram-positive bacteria. Here, we report the first in-depth analysis of the (p)ppGpp-regulon in an $\alpha$ proteobacterium using a high-resolution tiling array to better understand the pleiotropic stress phenotype of a relA/rsh mutant.
\end{abstract}

Results: We compared gene expression of the Rhizobium etli wild type and rsh (previously rel) mutant during exponential and stationary phase, identifying numerous (p)ppGpp targets, including small non-coding RNAs. The majority of the 834 (p)ppGpp-dependent genes were detected during stationary phase. Unexpectedly, 223 genes were expressed ( $\mathrm{p}$ )ppGpp-dependently during early exponential phase, indicating the hitherto unrecognized importance of (p)ppGpp during active growth. Furthermore, we identified two (p)ppGpp-dependent key regulators for survival during heat and oxidative stress and one regulator putatively involved in metabolic adaptation, namely extracytoplasmic function sigma factor EcfG2/PF00052, transcription factor CH00371, and serine protein kinase PrkA.

Conclusions: The regulatory role of (p)ppGpp in $R$. etli stress adaptation is far-reaching in redirecting gene expression during all growth phases. Genome-wide transcriptome analysis of a strain deficient in a global regulator, and exhibiting a pleiotropic phenotype, enables the identification of more specific regulators that control genes associated with a subset of stress phenotypes. This work is an important step toward a full understanding of the regulatory network underlying stress responses in $\alpha$-proteobacteria.

\section{Background}

Rhizobium etli is a soil-dwelling $\alpha$-proteobacterium that infects the roots of its leguminous host plant Phaseolus vulgaris, the common bean plant, in order to establish a nitrogen-fixing symbiosis [1-4]. Like most microorganisms in nature, $R$. etli primarily resides in a non-growing state in the soil, where it is confronted with diverse and stressful conditions, such as non-optimal temperatures and $\mathrm{pH}$ levels, near-starvation conditions and competition with other microbial populations [5]. Although growth is restricted, long periods of inactivity are

\footnotetext{
*Correspondence: jan.michiels@biw.kuleuven.be

Centre of Microbial and Plant Genetics, Katholiek Universiteit Leuven, Kasteelpark Arenberg 20, 3001 Heverlee, Belgium
}

sporadically interrupted by proliferation. This cycle of growth and starvation has been likened to a feast and famine lifestyle [6].

Sophisticated regulatory networks allow bacteria to sense and respond to a variety of environmental stresses to rapidly adjust their cellular physiology for survival. These networks comprise transcriptional regulators, sigma factors, proteases and small non-coding RNAs (ncRNAs) that interact in a complex manner in order to control the metabolic changes needed for adaptation [5]. The stringent response is a widespread global regulatory system, activated in response to various unfavorable growth conditions, and mediated by guanosine tetraphosphate (ppGpp) and guanosine pentaphosphate (pppGpp), collectively referred to as (p)ppGpp [7]. This alarmone coordinates

\section{() Biomed Central}


entrance into the non-growing state by inducing a general reprogramming of gene regulation, thereby downregulating cellular processes needed for growth and upregulating processes needed for survival. As a result, the available resources are diverted from growth to allow adaptation of the cell to the non-growing state $[8,9]$. The central role of this alarmone in the general stress response during the stationary phase is also illustrated by the increased sensitivity of (p)ppGpp-deficient mutants in various species to diverse stress factors [10]. Therefore, studying the (p) ppGpp regulon may be useful to identify novel regulators involved in the stress adaptation.

In Escherichia coli, the stress-induced alarmone production depends on two enzymes: RelA and SpoT [7]. When amino acids are limiting, uncharged tRNAs that bind ribosomes stimulate the ribosome-associated RelA to synthesize (p)ppGpp. Subsequent recovery when conditions are favorable again requires degradation of the alarmone, which is catalyzed by SpoT. SpoT is a bifunctional enzyme that can also synthesize (p)ppGpp in response to carbon, iron, phosphorus and fatty acid scarcity. Having two (p)ppGpp synthetases/hydrolases appears to be an exclusive feature of the $\gamma$-subdivision of the proteobacteria, as Gram-positive bacteria and most other Gram-negative bacteria, including $R$. etli, possess only a single RelA/SpoT homolog - usually referred to as Rel or Rsh - that displays both activities [10]. Most Gram-positive species additionally encode small proteins that consist solely of a synthetase domain [11].

(p)ppGpp primarily regulates gene transcription $[12,13]$. Several models have been proposed to accommodate the effects of (p)ppGpp on transcription. One of these models, the affinity model, argues for an increase in the availability of free RNA polymerase (RNAP) with increasing (p)ppGpp levels. As this alarmone binds near the active site of RNAP, the stability of the ribosomal RNA ( $r r n$ ) open complexes decreases. Consequently, (p) ppGpp will induce promoters with low RNAP affinity, such as cell maintenance and stress response genes $[14,15]$. In another model, the $\sigma$ factor competition model, the binding affinity of alternative sigma factors increases with increasing (p)ppGpp-levels compared to the housekeeping sigma factor $\sigma^{70}$. This results in a decrease of $\sigma^{70}$-bound RNAP and a downregulation of growth-related promoters that are dependent on high concentrations of $\sigma^{70}$-bound RNAP for maximal expression $[10,12,16]$. In addition to regulating sigma factor activity, (p)ppGpp is also required for sigma factor expression, as is the case for the stationary phase sigma factor $\sigma^{\mathrm{S}}$, the heat shock sigma factor $\sigma^{\mathrm{H}}$ and the sigma factor controlling nitrogen metabolism, $\sigma^{54}$, in $E$. coli $[17,18]$. Hence, these models for gene regulation of (p) ppGpp should be considered as working in concert. Finally, the recently identified cofactor DksA was demonstrated to stabilize binding of RNAP to (p) ppGpp, resulting in enhanced repression or stimulation of transcription in E. coli. However, the interaction between (p)ppGpp and DksA appears to be more complex as both factors also have independent and opposing effects on gene expression in E. coli $[13,19,20]$.

In agreement with (p)ppGpp's central role in stress adaptation, the alarmone was shown to be crucial in many complex physiological processes such as biofilm formation by Listeria monocytogenes, E. coli and Streptococcus mutans, development of multicellular fruiting bodies in Myxococcus xanthus and development of competence in Bacillus subtilis [10]. In addition, a fast growing number of reports demonstrate (p)ppGpp to be important during host interactions in diverse pathogens such as Vibrio cholerae, Pseudomonas aeruginosa, Legionella pneumophila, Francisella novicida, Enterococcus faecalis and Streptococcus pneumoniae [21-24]. Furthermore, various transcriptome studies showed that the alarmone (p)ppGpp is situated high up in the hierarchy of interconnected regulators in E. coli, controlling the expression and/or function of many other regulators such as Lrp, the cAMP receptor protein CRP, the integration host factor IHF, the flagellar master regulator FlhDC, the redox status sensing regulator ArcA and the morphogene BolA $[6,8,18,25,26]$.

(p)ppGpp also affects key aspects of the symbiosis between rhizobia and their leguminous host plants. In Sinorhizobium meliloti, a rsh mutant is defective in nodulation of Medicago sativa and overproduces the exopolysaccharide succinoglycan, which is crucial for root infection [27]. In $R$. etli, (p)ppGpp controls the physiological adaptation of the bacterium to the endosymbiotic state [28,29]. Although the $r s h$ mutant induces nodulation, the bacteroids are morphologically different compared to the wild type, and nitrogen fixation activity is drastically reduced. Several nitrogen fixation and quorum-sensing genes, essential for symbiosis, were shown to be part of the alarmone regulon, including the symbiotic $\sigma^{\mathrm{N}}$ that is required for expression of nitrogen fixation genes [29]. Recently, a detailed phenotypic analysis of the $r s h$ (previously referred to as $\mathrm{relA}$ or $\mathrm{rel}_{\mathrm{Ret}}$ ) mutant showed a prominent role for the alarmone in the general stress response of $R$. etli during free-living growth and symbiosis [30].

In order to obtain new insights into the molecular basis of adaptation of $R$. etli to unfavorable growth conditions, we performed a genome-wide transcriptome analysis to compare global gene expression between the wild type and a rsh mutant during different free-living growth phases.

This study is the first in-depth analysis of (p)ppGppdependent gene regulation in an $\alpha$-proteobacterium, revealing notable differences from the well-studied role 
of (p)ppGpp in E. coli. Of the many detected (p)ppGpp targets that may contribute to the observed stress phenotypes of the rsh mutant, we performed a phenotypic analysis of three specific previously uncharacterized regulators, that is, sigma factor EcfG2/PF00052, DNA-binding transcription factor $\mathrm{CH} 00371$ and serine kinase PrkA/CH02817. Our results show that the stress phenotypes of mutants lacking EcfG2 or CH00371 correspond to a subset of the rsh mutant phenotypes, while PrkA may be involved in metabolic adaptation. In addition, we identified several upstream and downstream elements in the stress response pathways of these three novel (p)ppGpp-dependent regulators, providing added detail to the complex picture of the role of (p)ppGpp in R. etli.

\section{Results and Discussion}

\section{Experimental design of the transcriptome analysis}

Previously, we reported on the crucial role of (p)ppGpp during symbiosis and free-living growth in $R$. etli CNPAF512 using a rsh mutant $[29,30]$. Based on these findings, we decided to carry out a transcriptome analysis to characterize to what extent (p)ppGpp deficiency affects gene expression in $R$. etli. The intracellular (p) ppGpp content of the $R$. etli wild type, $r s h$ mutant and complemented rsh mutant was determined previously [29], showing the rsh mutant to be (p)ppGpp-deficient. However, due to the sensitivity of the assay, the presence of trace amounts of (p)ppGpp in the rsh mutant, possibly resulting from the presence of an as yet unidentified synthetase gene, cannot be ruled out.

At the time of the experimental setup, only the genomic DNA sequence of $R$. etli CFN42 was available [31]. Therefore, a custom whole-genome microarray for $R$. etli CFN42 as well as a CFN42-derived rsh mutant was constructed. Phenotypic analysis of this mutant showed that a lack of (p)ppGpp results in an extended lag phase in different media, an altered morphology and a $75 \%$ reduction of nitrogen fixation activity in plants inoculated with the CFN42 rsh mutant compared to the wild type (data not shown). All phenotypes could be fully complemented by providing rsh of CNPAF512 in trans and are in agreement with our previously published rsh mutant analyses [29,30].

To determine the role exerted by the alarmone (p) ppGpp in the regulation of transcription during free-living growth and growth arrest of $R$. etli, total RNA samples were taken at three different time points corresponding to early and late exponential and stationary phase, respectively (Additional file 1).

\section{Global overview of gene expression}

The $R$. etli CFN42 genome contains 6,030 annotated protein-encoding genes, 67 pseudo genes, 3 rRNA operons and 50 tRNA genes. Recently, we described an additional 89 ncRNA genes [32]. In both the wild type and $r s h$ mutant, over $97 \%$ (or $(683+870) / 1,593)$ of proteinencoding genes that are transcribed above the detection limit (see Materials and methods) during early exponential growth are also expressed during late exponential growth. In addition, numerous genes are induced in the course of growth, as $20 \%$ (or $(157+227) / 1,937$ ) of the genes expressed in late exponential phase are not transcribed during early growth (Figure 1).

We identified a large number of differentially expressed genes during exponential and stationary phase, both (p)ppGpp-dependent and independent, the former being consistent with the role of $r s h$ as a global regulator described in other species [33-35]. The extent of differential expression is illustrated by the ratio/intensity MA plots in Additional file 2. Alarmone dependency was determined by comparing gene expression of the wild type and rsh mutant during each of the three sampled growth phases (Figure 2a). A total of 834 (p) ppGpp-dependent genes with an expression ratio of at least two-fold were found. Approximately half of these genes (520) were expressed exclusively during stationary

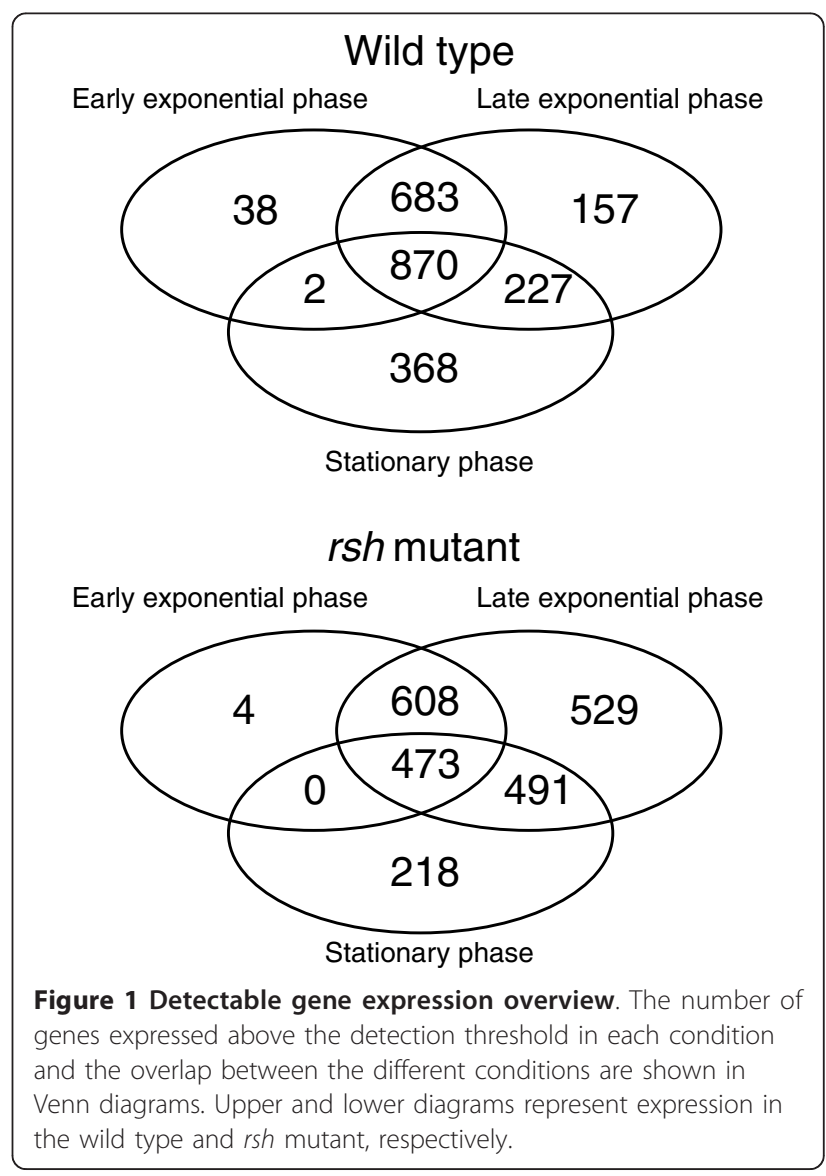


(a)

\section{(p)ppGpp-dependent genes}

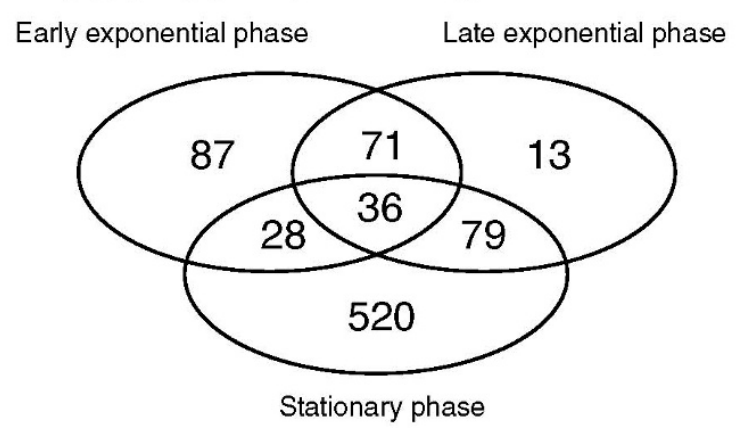

(b) Stationary phase genes

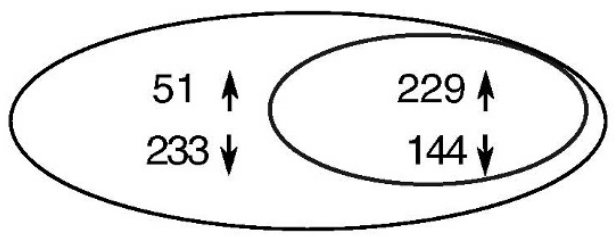

Figure 2 (p)ppGpp-dependent gene expression. (a) Venn diagram of all differentially expressed (p)ppGpp-dependent genes during early exponential phase, late exponential phase and stationary phase. (b) Venn diagram of all genes expressed during stationary phase (large ellipse). The overlap with (p)ppGppdependent genes (see (a)) shows all (p)ppGpp-dependent stationary phase genes (small ellipse). Upwards and downwards oriented arrows indicate gene induction and repression, respectively.

phase and only a minority (36) were found to be (p) ppGpp-dependent during all growth conditions.

By comparing expression in the wild type during stationary and early exponential phase, we identified 657 stationary phase genes (Figure $2 \mathrm{~b}$ ), representing $11 \%$ (or $657 / 6,030)$ of the annotated protein-coding genes. The overlap of (p)ppGpp-dependent genes and stationary phase genes shows that just over half $(57 \%$ or $(229+$ 144)/657) of stationary phase genes are (p)ppGppdependent. Because $61 \%$ (or 229/373) of these were upregulated (Figure 2b), the alarmone (p)ppGpp seems to have a primarily inducing role in $R$. etli. A comparable number of (p)ppGpp-dependent genes were found in other bacteria: 490 (11\%) of all genes in E. coli and 194 (6\%) in Corynebacterium glutamicum after (p) ppGpp-induction by serine hydroxymate [18,34], 589 genes (7\%) upon induction of (p)ppGpp synthesis in Streptomyces coelicolor [35] and 373 (18\%) of all genes after treatment with mupirocin in Streptococcus pneumoniae [23].

The microarray data were confirmed by analyzing the expression levels of 14 representative genes using reverse transcription-quantitative PCR (RT-qPCR; see Materials and methods). For each gene, expression during early exponential phase and stationary phase in the wild type and rsh mutant was measured so that three different ratios could be plotted versus the respective ratios obtained by microarray analysis (Figure 3), showing the array data to be in good agreement with the RTqPCR data.

\section{The effect of (p)ppGpp on global gene expression during} stationary phase

During stationary phase in E. coli, the alarmone (p) ppGpp induces a downregulation of processes involved in cell growth, such as DNA replication and translation, and an upregulation of specific metabolic pathways to cope with certain nutrient deficiencies as well as general stress responses to protect the cell against immediate and future harmful conditions. In order to better understand the role of (p)ppGpp in the global reprogramming of $R$. etli's transcriptome, we compared the expression of wild type and rsh mutant during stationary phase. As samples were taken approximately 6 hours after growth arrest, the observed differences in expression include both direct and indirect effects caused by a lack of alarmone. Of the 663 differentially expressed genes, 292 and

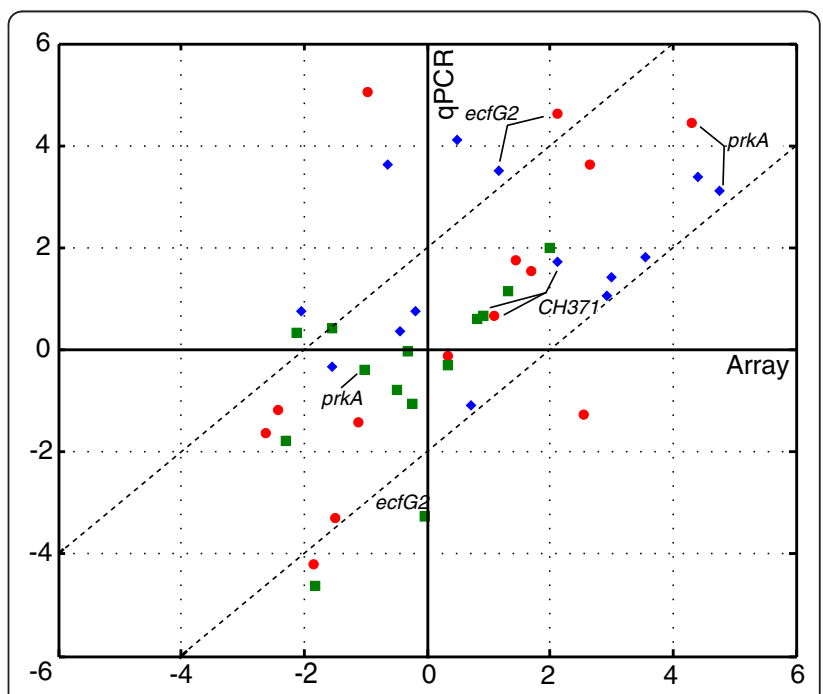

Figure 3 RT-qPCR validation of the microarray data. Expression of 14 genes was determined using RT-qPCR for the wild type and rsh mutant in early exponential phase and stationary phase. The $\log _{2}$-transformed mean values of two biological replicates were used to report three different fold changes for each gene (Y-axis) compared to the respective microarray fold changes ( $X$-axis). See Additional file 6 for a complete list of the plotted fold change values. Red dots, wild type stationary phase versus early exponential phase. Blue diamonds, wild type versus rsh mutant in stationary phase. Green squares, wild type versus rsh mutant in exponential phase. The fold changes for ecfG2/PF00052, CH00371 and prkA/ $\mathrm{CH} 02817$ are indicated. 
371 were upregulated and downregulated, respectively, in the wild type compared to the rsh mutant (Figure $2 \mathrm{a})$. These genes were further grouped based on predicted functional role and category (Additional file 3). An overview of the functional categories (Figure 4a) shows the mutant to be less well adapted to the nongrowing lifestyle as more growth-associated genes, involved in cell wall biosynthesis, energy production and intracellular trafficking and secretion, are induced in the mutant. Notably, the replication and recombination category is strongly represented in the mutant due to the high number of insertion sequence (IS)-related genes that show expression. An equal number of genes with unknown function were up- and downregulated. In the following paragraphs, selected functional categories, primarily focused on regulation and possible links with the pleiotropic stress phenotype, will be discussed in more detail.

\section{Transcriptional regulators and signal transduction}

The link between changes in extracellular conditions and concomitant adaptation of genome expression involves a combination of sensors, transporters, phosphorylation cascades and the modulation of transcription factors [36]. Most of these belong to the 'transcription' and 'signal transduction' categories, of which 29 and 26 genes are differentially expressed, respectively, in the wild type compared to the (p)ppGpp-deficient mutant at onset of growth arrest (Additional file 3).

By clustering the differentially expressed genes of these two categories, we identified two main groups (Figure 5). The first group contains genes that are under negative (p)ppGpp control during primarily the stationary phase and include the LysR transcriptional regulators nocR and nodD3, the two-component sensor kinase $\operatorname{vir} A$ and two diguanylate cyclases, PD00137 and PE00107. The second group contains genes that are under positive (p)ppGpp control during primarily the stationary phase, encoding among others the transcriptional regulators RirA and BolA-like $\mathrm{CH} 02287$, the CarD-like regulator $\mathrm{CH} 04025$, the two-component response regulators $\mathrm{CH} 02556$ and $\mathrm{CH} 03335$, and the $\mathrm{N}$ acyl-L-homoserine lactone (AHL) synthase CinI.

Several of these transcriptional regulators have previously been shown to play a role in the adaptation to adverse conditions in other species and can partly explain the pleiotropic stress phenotype of the rsh mutant. In E. coli, BolA controls expression of a number of cell wall proteins, is partially responsible for the

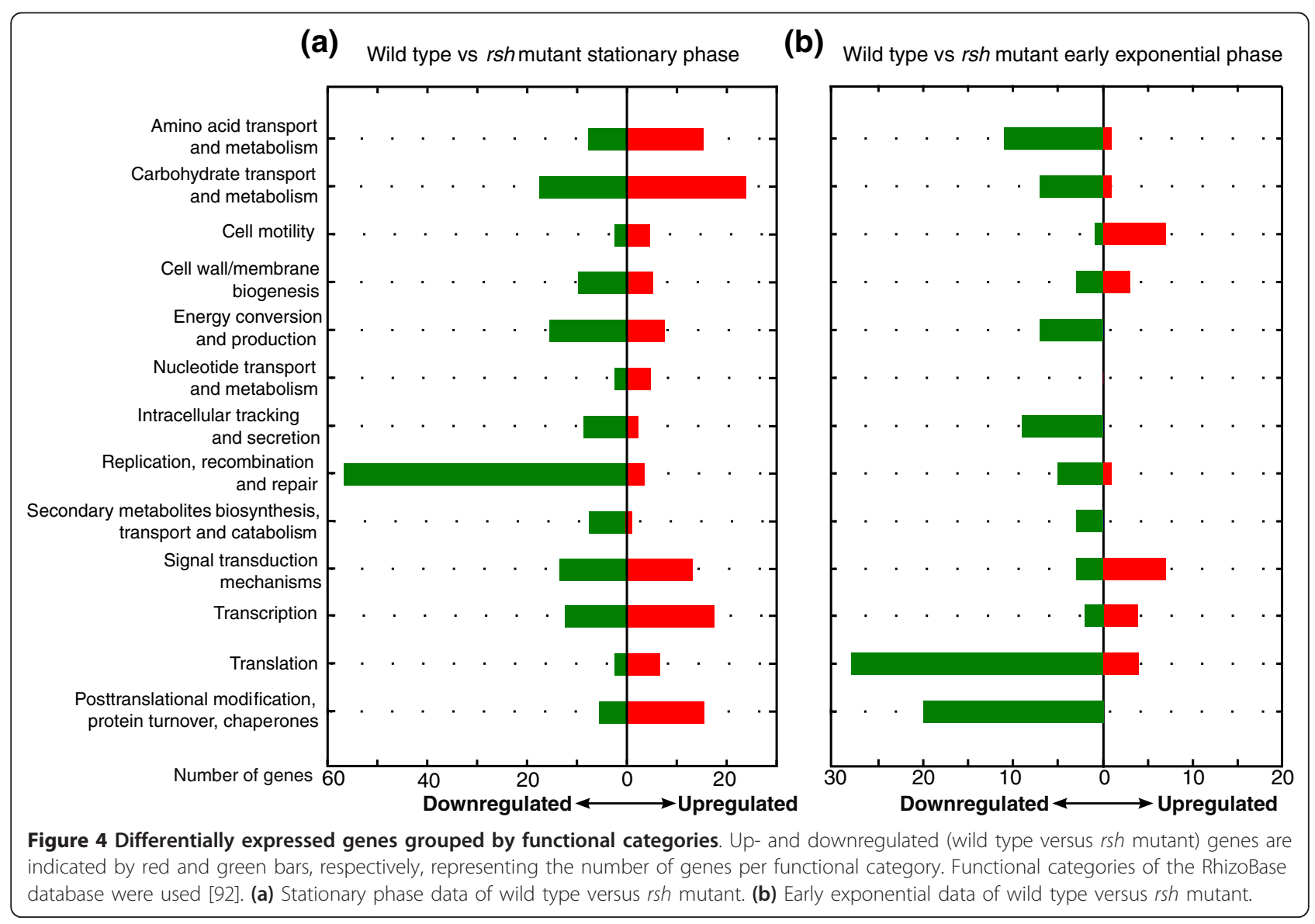




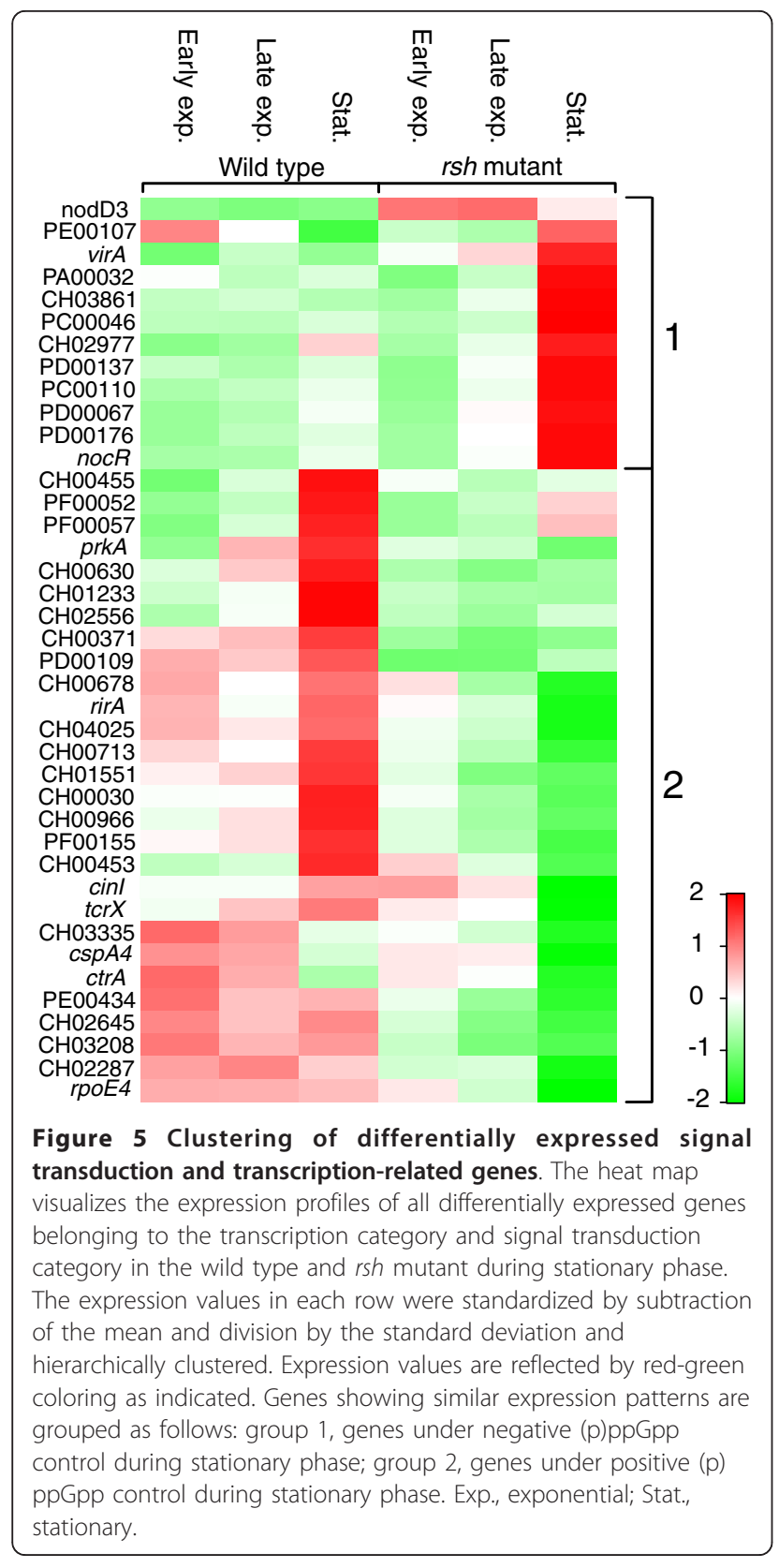

coccoid morphology of stationary phase cells and is also expressed in a (p)ppGpp-dependent manner, being under control of RpoS $[5,18,33]$. Reduced BolA levels may therefore contribute to the altered morphology of the $R$. etli rsh mutant. Furthermore, expression of the global iron-responsive regulator RirA that controls the synthesis of heme, FeS-clusters and bacterioferritin in rhizobia, is under positive (p)ppGpp control as well $[37,38]$. Accordingly, expression of bacterioferritin ( $b f r)$ was positively upregulated in the $R$. etli wild type, suggesting that (p)ppGpp contributes to iron homeostasis. Conversely, a lack of iron may cause an increase in the level of (p)ppGpp in order to regulate iron homeostasis in the cell as reported in E. coli and B. subtilis [39,40]. Since iron plays a crucial role in the oxidative stress response, incomplete iron sequestration may also contribute to the increased oxidative stress sensitivity of the rsh mutant [30,41]. Other regulators under positive (p) ppGpp control include two members of the cold shock protein family (CspA3, CspA4), a putative member of the UspA family (CH01233), the SOS response regulator LexA and the two-component regulator TcrX. TcrX is orthologous to PhyR of Methylobacterium extorquens, which regulates many stress response genes and was shown to play a role in the osmotic stress response in $R$. etli as well $[42,43]$.

\section{Sigma factors}

R. etli CFN42 possesses 23 sigma factors that determine the promoter specificity of the RNAP holoenzyme by binding to the core enzyme. Therefore, differential expression and/or activity of sigma factors can redirect global gene expression. During exponential growth, transcription is largely under control of the housekeeping sigma factor $\sigma^{70}$ as its binding affinity for RNAP and intracellular concentration are much higher compared to the other sigma factors. These alternative sigma factors have specific regulons and will redirect transcription upon unfavorable conditions. Bacteria like $R$. etli that have a complex lifestyle or encounter diverse environmental conditions usually display an increased number of sigma factors $[5,44]$.

Upon transition to stationary phase, the reversible switch to a less $\sigma^{70}$-dominated expression in E. coli is accomplished not solely by (p)ppGpp but also by DksA and the anti- $\sigma^{70}$ factor Rsd. In $R$. etli, expression of $d k s A$ is reduced over eight-fold in stationary phase compared to early exponential phase in a (p)ppGpp-independent manner. The role of DksA in $\alpha$-proteobacteria is so far unknown. Furthermore, no Rsd homolog is found in $R$. etli or other other $\alpha$-proteobacteria. $R$. etli may compensate for the lack of a specific anti- $\sigma^{70}$ factor, as we observed a (p)ppGpp-independent drop in expression of the housekeeping sigma factor sigA to below the detection limit while expression in E. coli of $\sigma^{70}$ remains constant during stationary phase.

Of all the alternative sigma factors, only the extracytoplasmic function (ECF) sigma factor PF00052 was upregulated at least two-fold during stationary phase compared to early exponential phase in the wild type. The ECF sigma factor rpoE4 is expressed at the same level during all conditions in the wild type, but dropped below the expression threshold during stationary phase in the rsh mutant. Consequently, two ECF sigma factors, PF00052 and rpoE4, were upregulated over two-fold in the wild type compared to the rsh mutant during stationary phase (Figure 5). In E. coli, only the level of the 
stationary phase sigma factor $r p o S\left(\sigma^{\mathrm{S}}\right)$ increases with (p)ppGpp concentration and plays a crucial role as global regulator in the (p)ppGpp-dependent stress response $[45,46]$. In contrast, $\varepsilon$ - and $\alpha$-proteobacteria, including $R$. etli, lack such a stationary phase sigma factor and, so far, it is unclear which system takes over this function.

Our data suggest that both PF00052 and RpoE4 may be important sigma factors in $R$. etli adaptation to stationary phase and possibly fulfill a role similar to RpoS in E. coli. First, both sigma factors are expressed during stationary phase. Second, both sigma factors are the most highly upregulated (p)ppGpp-dependent alternative sigma factors during stationary phase. Third, both share considerable sequence similarity and were recently classified in a group of proposed general stress response sigma factors that is exclusively found in $\alpha$-proteobacteria [47]. Fourth, it was recently shown in $R$. etli that RpoE4 regulates gene expression in response to several stress conditions including oxidative, saline and osmotic stress. Fifth, we found that PF00052 is also involved in the (p)ppGpp-dependent stress response and is in part functionally redundant with RpoE4 (see below).

Transcriptome analysis of an $R$. etli rpoE4 mutant and overexpression strain revealed 98 genes to be regulated by this sigma factor [42]. Since transcription of rpoE4 is (p)ppGpp-dependent, we investigated to what extent the reported RpoE4 regulon is (p)ppGpp-dependent. In total, 60 of the 98 genes belonging to the reported regulon are differentially expressed in our data (Additional file 4). Of these genes, $82 \%$ are (p)ppGpp-dependent and $92 \%$ are up- or downregulated during stationary phase compared to early exponential phase in the wild type. Upon rpoE4 overexpression, $74 \%$ of the reported upregulated genes were found to be (p)ppGpp-dependent.

Considering the RpoE4-regulated genes, all 16 genes predicted to encode proteins associated with cell envelope biogenesis are also (p)ppGpp-dependent. Similarly, E. coli's sole ECF sigma factor, $\sigma^{\mathrm{E}}$, regulates many genes involved in the biogenesis and stress response of the cell envelope $[48,49]$. Other RpoE4 and (p)ppGpp-dependent genes include a putative Mn-catalase (CH00462), a putative pyridoxine-phosphate oxidase (CH03474), an alphaglucoside $\mathrm{ABC}$ transporter (algE), and a CarD-like transcriptional regulator (CH04025). The latter is a crucial regulator in Mycobacterium tuberculosis that is upregulated in response to oxidative stress, DNA damage and starvation $[48,49]$. The above suggests that the pleiotropic stress phenotype of the $R$. etli rsh mutant can be explained, at least in part, by downregulation of (p) ppGpp-dependent sigma factors that play a crucial role in orchestrating the stress response.

\section{Non-coding RNAs}

Our data indicate that (p)ppGpp controls expression of many protein-coding genes. In addition, we identified 33 alarmone-dependent ncRNAs expressed during stationary phase. Of these, 28 were positively regulated by (p) ppGpp, including one glycine riboswitch, 17 novel ncRNAs, 4 previously identified but uncharacterized ncRNAs, and the 6 well characterized ncRNAs (6S RNA, tmRNA, signal recognition particle 4.5S RNA, RNase P, and ctRNA of plasmids p42d and p42e) (Additional file 5). Only five ncRNAs, all novel, were negatively regulated by (p)ppGpp.

So far, no ncRNAs have been reported to be (p) ppGpp-dependent in any organism. However, in recent years, an increasing number of ncRNAs have been found to be regulated by alternative sigma factors in $E$. coli, Salmonella enterica serovar Typhimurium, $L$. monocytogenes, B. subtilis and S. coelicolor [50-53]. Therefore, the (p)ppGpp-dependent ncRNAs of $R$. etli could be regulated by alternative sigma factors as well. Additionally, ncRNAs can also regulate sigma factors, as is the case for $\sigma^{\mathrm{S}}$ in $E$. coli whose translation is regulated by DsrA and RprA [50-53].

The level of 6S RNA was almost 14-fold lower in the alarmone-deficient mutant during stationary phase in $R$. etli. This is unlike in E. coli, where 6S RNA is not under (p)ppGpp control either in vitro or in vivo [54,55]. However, 6S RNA transcription appears to be complexly regulated in $E$. coli as several stress regulators, such as Fis, H-NS, Lrp and StpA, were shown to be inhibitors under in vitro conditions [55]. Recently, transcriptional analysis of a 6S RNA-deficient mutant showed 273 genes to be differentially expressed during stationary phase. Surprisingly, loss of 6S RNA in E. coli also resulted in an increase of the basal (p)ppGpp level mediated by an altered activity of SpoT and not RelA [56]. Therefore, 6S RNA is clearly embedded in stationary phase adaptation, although its association with (p) ppGpp in R. etli and E. coli may differ.

Expression of bacterial RNase P and tmRNA was almost 13- and 6-fold downregulated, respectively, in the $R$. etli rsh mutant compared to the wild type. Although the synthesis and processing of tRNA is expected to be downregulated during growth arrest, 38\% of the tRNAs were upregulated in the wild type during stationary phase compared to early exponential phase and $56 \%$ of the tRNAs were upregulated in an alarmone-dependent manner. The upregulation of bacterial RNase P during stationary phase in an alarmone-dependent manner is in line with the unexpected upregulation of several tRNAs as RNase $\mathrm{P}$ is required to process the 5 ' end of precursor tRNAs. Expression of tmRNA is also upregulated in $R$. etli. This alarmone-dependence of tmRNA expression has not been reported in E. coli, although a lack of $6 \mathrm{~S}$ RNA results in a three-fold higher expression of the SmpB protein, which acts together with tmRNA [56]. However, the expression level of 
tmRNA was not reported. Interestingly, the 6S RNA mutation is compensated for by an increase of the basal (p)ppGpp level, which indicates that the tmRNA/SmpB system might be alarmone-dependent in E. coli also. Still, (p)ppGpp is not needed for mRNA cleavage in the A site of the ribosome by tmRNA [57]. In contrast, both tmRNA and $s m p B$ of Streptococcus pyogenes were shown to be upregulated in a relA-independent amino acid starvation response [58].

\section{Translational apparatus}

In addition to inducing general stress and nutrient scavenging regulons, the accumulation of (p)ppGpp upon growth arrest in $E$. coli is characterized by a stringent downregulation of expression of the translational apparatus as a mechanism to fine-tune the metabolically expensive process of protein synthesis according to the growth state of the cell $[9,59]$. As expected, during the stationary phase all 56 genes encoding ribosomal proteins were downregulated in the $R$. etli wild type compared to the exponential phase. However, nearly all (53 out of 56) of these were downregulated in the rsh mutant as well. Although this (p)ppGpp-independent downregulation is in conflict with the established $E$. coli paradigm of the stringent response, a similar response was described in a rel mutant of Corynebacterium glutamicum upon addition of serine hydroxamate [9,34,59]. Therefore, the difference in transcriptional regulation of ribosomal protein expression during growth arrest suggests that the stringent response in $R$. etli may deviate from the classical model in E. coli.

Other genes encoding parts of the translational machinery that were positively regulated by (p)ppGpp in $R$. etli include the homolog of E. coli yhbH (CH00406) and two EF-Tu elongation factors (tufA, tufB). In E. coli, $\mathrm{YhbH}$ is involved in the temporary storage or dimerization of ribosomes during stationary phase. This process was shown to contribute to the survival of E. coli [28]. In accordance with our data, the YhbH ortholog of $B$. subtilis $(y v y D)$ is also under positive (p)ppGpp control [60]. However, in contrast to the positive (p)ppGppdependent regulation of tufA and tufB in $R$. etli, TE-Tu factors in $E$. coli and B. subtilis were previously shown to be under negative control of (p)ppGpp $[8,60]$. Interestingly, translation factors such as TE-Tu are GTPases that can bind (p)ppGpp and associate with the ribosome, indicating that they may have a downstream role in (p)ppGpp-dependent gene regulation [13].

\section{Post-translational modification, repair and recombination}

The (p)ppGpp-dependent stress adaptation during stationary phase involves 20 genes belonging to the posttranslational modification category, of which 15 were positively regulated. These include several components of the ATP-dependent Clp protease system, such as $\operatorname{clp} X, \operatorname{clpP} 2, \operatorname{clpP} 3, \operatorname{clp} A$ and $\operatorname{clpS}$, as well as the ATP- dependent proteases lon and ftsH [61]. These proteases allow cells to cope with misfolded or denatured proteins, the abundance of which increases during stress conditions, such as heat stress, in order to prevent protein aggregation and to enable recycling of amino acids [5]. A similar (p)ppGpp-dependent regulation was observed for $\operatorname{clp} A$ in $E$. coli as well as $\operatorname{clpP} 1$ and $c l p C$ in C. glutamicum [33,34]. Thus, the (p)ppGpp-dependent increase of tmRNA in $R$. etli correlates with the increase in proteases as the Clp system and Lon are needed to degrade tmRNA-tagged polypeptides in E. coli [62].

Proteases and chaperones are also involved in regulating transcriptional regulators and other growth-phase regulated proteins, such as RpoS, Dps and GlnA in $E$. coli [63]. Therefore, by controlling proteolysis, the alarmone (p)ppGpp mediates the cellular reprogramming of $R$. etli at the post-transcriptional level as well. Other positively controlled genes include the probable serine protease $\mathrm{CH} 01273$, the small heat shock protein PF00472 as well as genes required to cope with oxidative stress, such as osm $C$ and $g r l A$.

Rather unexpectedly, very few genes of the repair and recombination category were under positive stringent control. However, several IS-related genes were negatively controlled by (p)ppGpp, including 47 transposases, one resolvase, and one integrase. Therefore, these data suggest that the alarmone may assist in repressing insertional activity and mobility of IS-related elements.

\section{Other processes}

The impact of (p)ppGpp as a global regulator of transcription is further illustrated by its control of genes involved in diverse cellular processes. In E. coli, the alarmone plays a central role in restructuring metabolism upon nutrient starvation and growth arrest, thereby increasing the range of active metabolic pathways and nutrient scavenging potential [33]. In $R$. etli, the alarmone likely has a similar role in metabolism as differential gene expression was detected for 22 genes involved in amino acid metabolism, 41 genes in carbohydrate metabolism, 9 in lipid metabolism and 22 in energy production.

(p)ppGpp was shown in E. coli to induce amino acid biosynthesis pathways depending on the availability of limiting amino acids. However, compared to exponential phase, no clear upregulation during stationary phase of one or more specific amino acid pathways was found in the $R$. etli wild type. Only a few genes involved in amino acid metabolism were positively controlled by (p) ppGpp (phhA, cysE1, glnA2, trpE). In addition, 13 amino acid synthesis genes $(\operatorname{trp} F, \operatorname{trp} A, \operatorname{his} B, \operatorname{asn} B, \operatorname{aro} Q 1$, aroF, ilvI, aatA, lys $C$, argG2, tyrA, leuD, asd) along with the P-II regulator $g \ln B$, which regulates glutamine synthetase in response to nitrogen levels, were downregulated in a (p)ppGpp-independent manner during 
stationary phase compared to exponential phase. Therefore, during stationary phase, amino acid biosynthesis in $R$. etli is downregulated rather than upregulated as in $E$. coli.

Several genes encoding key enzymes of carbohydrate metabolism were induced by (p)ppGpp, including the transaldolase tal of the pentose pathway, $g \operatorname{lgC}$ involved in starch and sucrose metabolism, the glycolytic gene $f b a B$ and the gene encoding trehalose-6-phophatase, $o t s B$. These genes were also shown to be under positive control by (p)ppGpp in E. coli upon amino acid starvation [33]. FbaB is a fructose-bisphosphate aldolase whose reaction product can exert feedback control on the glycolytic flux and is also required for ribosome recycling during carbon starvation [6]. Moreover, OtsB produces the disaccharide trehalose from trehalose-6phosphate, which is produced by OtsA using UDP-glucose and glucose-6-phosphate. Not only is trehalose an energy and carbon source, it also stabilizes and protects proteins and membranes from dehydration, oxidation and cold [64]. Recently, it was shown that all three trehalose synthesis pathways known to date are present in $S$. meliloti. However, only the OtsA pathway is important for osmo-inducible trehalose synthesis [65]. In $R$. etli, overexpressing ots $A$ improves symbiotic efficiency and drought tolerance of its host $P$. vulgaris [66]. During stationary phase, ots $A$ and ots $B$ have a different expression pattern; ots $B$ is induced by (p)ppGpp while ots $A$ is constitutively expressed in the wild type but under negative (p)ppGpp control upon growth arrest. It is possible that this (p)ppGpp-dependent regulation of trehalose synthesis contributes to the previously observed increased sensitivity of the rsh mutant to osmotic stress [30].

The link between the stringent response and the available carbon sources remains unclear. In E. coli, the (p) ppGpp synthetase/hydrolase SpoT interacts with acyl carrier proteins (ACPs) of fatty acid metabolism [67]. $R$. etli contains four acyl carrier proteins, of which only two $(a c p P, a c p X L)$ were expressed during growth and downregulated upon growth arrest independently of (p) ppGpp. In contrast to E. coli, no clear (p)ppGpp-dependent regulation of lipid metabolism genes was observed. Also, most of the nucleotide biosynthesis genes are downregulated during stationary phase compared to exponential phase in the wild type, reflecting the decreased need for nucleotides. Only six nucleotide biosynthesis genes were found to be under control of (p) ppGpp in $R$. etli. This is in accordance with the observed (p)ppGpp-independent downregulation of ribosomal proteins.

As well as regulating $R$. etli's biosynthetic potential, (p) ppGpp also controls its transport capacity during stationary phase. Twenty-one genes related to $\mathrm{ABC}$ transporters were under positive (p)ppGpp control, such as $p o t F, d p p A$, proX, aglK, and $g g u B$, while 12 were repressed. Most of these transporters allow for the uptake of amino acids, peptides and monosaccharides. In addition, two secretion-associated genes ( $\sec B$ and pilA) were upregulated and seven genes involved in type IV secretion were downregulated (virB1a, 2a, D4, B6a, $B 8 a, B 8 d, B 10)$. Interestingly, the pilin subunit pilA was the most highly expressed protein-encoding gene in $R$. etli during stationary phase.

Energy production drops during the stationary phase as more than 25 genes predicted to be involved in oxidative phosphorylation were downregulated compared to exponential phase in the wild type. In contrast to $E$. coli, $76 \%$ of the differentially expressed genes that belong to the energy production category, 95 in total, are not under (p)ppGpp control [33]. During free-living growth, $R$. etli uses cytochrome $\mathrm{aa}_{3}$ terminal oxidases, encoded by ctaCDGE, coxPONM and CH00981$\mathrm{CH} 00985$ [31,68]. The ctaCDGE terminal oxidase was downregulated during stationary phase in both the wild type and the rsh mutant. On the other hand, the coxPONM alternative terminal oxidase was upregulated during stationary phase in the wild type but not in the $r s h$ mutant. The third probable terminal oxidase was not expressed. Therefore, the alternative terminal oxidase coxPONM is likely to play an important role during (p)ppGpp-dependent stationary phase adaptation.

In addition to the decrease in energy production, flagellum synthesis and motility is also downregulated during stationary phase, reflecting that it is a highly energy demanding process. In $E$. coli, flagellar genes are under positive (p)ppGpp control $[18,19,69]$. Similarly in $R$. etli, (p)ppGpp positively regulates flagellar gene expression. However, this regulation occurs primarily during the exponential phase instead of the stationary phase, as 25 of the 35 flagellar genes were expressed above threshold during the exponential phase compared to 10 during the stationary phase. Of the latter, three flagellar hookrelated genes $(f l g D, f l g E, f l g L)$ and two flagellin synthesis regulators $(f l a F, f l b T)$ were upregulated in the wild type compared to the rsh mutant [70]. FlgD forms a scaffold on which the hook subunit FlgE polymerizes on the envelope-embedded rod to form the flexible hook structure. FlgL is a junction protein connecting the rigid flagellar filament. In short, (p)ppGpp regulates several crucial flagellar genes in $R$. etli.

\section{The effect of (p)ppGpp on global gene expression during early exponential phase}

By comparing the expression data of the wild type and $r s h$ mutant during early exponential growth, we identified 203 differentially expressed genes, of which 59 were under positive stringent control and 144 under negative 
stringent control. This is surprising as transcription during the exponential phase of a (p)ppGpp-deficient mutant and the wild type is generally thought to be very similar. The alarmone is considered to be a stationary phase or growth arrest-specific messenger that switches the cellular metabolism to a non-growing state. During favorable growth conditions, (p)ppGpp is produced at a low basal level and rapidly accumulates in response to growth-perturbing conditions. Furthermore, during the exponential phase, a (p)ppGpp-deficient mutant of $E$. coli is phenotypically very similar to the wild type, although a decreased growth phase-independent thermotolerance has been reported [71]. However, to the best of our knowledge, to this date no detailed comparison of global transcription during exponential growth has been described for a wild type and relA spoT mutant of $E$. coli, which serves as the stringent response model organism. Still, it was recently shown that almost 300 genes were differentially expressed in an rpoS mutant of E. coli during exponential growth, even though RpoS is known as the stationary phase sigma factor [72]. This would suggest that a difference in expression during logarithmic growth in a (p)ppGpp-deficient mutant could be expected as (p)ppGpp regulates the expression and activity of RpoS in E. coli. Moreover, a major difference in expression during growth in the absence of (p) ppGpp was also previously observed in M. tuberculosis and C. glutamicum [34,73]. Our data are in agreement with these reports, showing that the low basal level of (p)ppGpp is functionally relevant during active growth. This additional function is also in agreement with the observed increase in sensitivity to several acute and chronic stresses of a $R$. etli rsh mutant during exponential growth [30].

A comparison of the (p)ppGpp-dependent genes during early exponential phase and stationary phase showed that only 50 genes were differentially expressed in both states. Of this fraction, only half of the genes showed similar positive or negative control during both phases. This suggests that the function of (p)ppGpp differs during active growth and growth arrest, possibly through involvement of other regulators. To further understand the impact and role of the alarmone during exponential growth, we again grouped the up- and downregulated genes in functional categories (Figure 4b). As 71\% of these genes were under negative (p)ppGpp regulation, the alarmone plays a primarily repressing role during logarithmic growth, in contrast to the observed predominantly inducing role upon growth arrest. For example, the alarmone induces 19 transporters during stationary phase while it represses 12 during early exponential phase. Other genes under negative (p)ppGpp control include 10 conjugal transfer proteins, 5 IS-related transposases and 26 ribosomal proteins. In contrast, nine motility genes were upregulated by (p)ppGpp, such as three of the four basal-body rod proteins (flgBCG), one of the three flagellar switch proteins that interact with the chemotaxis system $(f l i N)$ and three chemotaxis proteins (motA, cheW5, cheY1). Therefore, (p)ppGpp has a similarly inducing role on flagellar genes during growth as observed upon growth arrest. A swimming test on $0.2 \%$ agar plates corroborates this observation (Figure 6). The (p)ppGpp-deficient mutant showed reduced swimming activity compared to the wild type, a phenotype that could be partially complemented by providing the $r s h$ gene in trans. Hence, the alarmone is required for optimal motility, as was also previously reported for $E$. coli [19,69].

Remarkably, in addition to growth-related genes, many post-translational modification genes were under negative stringent control in the rsh mutant. These comprise numerous chaperones, including the three major ones, tig, dnaK and groEL-groES, involved in folding of new proteins as well as in proper assembly of unfolded proteins and refolding of misfolded proteins generated under stress conditions [74]. DnaK is also involved in chromosomal DNA replication and is part of the osmotic stress response, in addition to osm $C$ [74]. Other upregulated heat shock proteins in the $r s h$ mutant include four peptidases ( $h s l V$, lon, traF, htpX2) and one protease $(f t s H)$. Although exponentially growing cells are considered to be less stressed, this increased expression of many heat shock proteins in actively growing cells in the absence of (p)ppGpp might indicate a defect or disruption in protein homeostasis, rather then merely an increase in translational activity. Therefore, this stress response during growth is in accordance with increased stress sensitivity of the rsh mutant as observed previously [30].

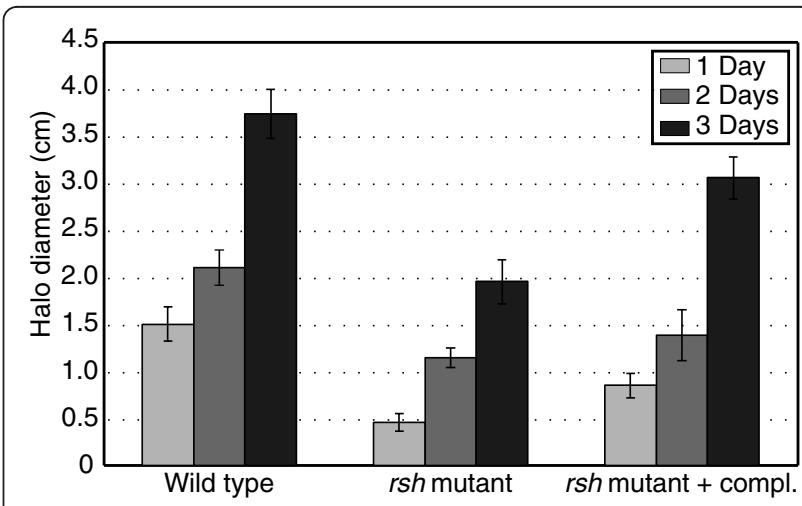

Figure 6 Swimming motility test. Swimming halo diameter observed on $0.2 \%$ agar TY plates on three consecutive days for wild type, rsh mutant and complemented rsh mutant. The mean values and standard deviation of five biological replicates are shown. The differences over time are statistically significant between the different strains $(P<0.001)$. 


\section{Functional analysis of three (p)ppGpp-dependent regulators}

To gain insight into the (p)ppGpp-controlled adaptation of $R$. etli to the stationary phase and diverse stresses as previously reported [30], we selected three different types of previously uncharacterized regulators based on their strongly (p)ppGpp-dependent expression during stationary phase and belonging to the group of transcriptional regulators (ecfG2, phrR) or signal transduction $(p r k A)$. The microarray expression patterns of these regulators were confirmed using RT-qPCR (Figure 3). A phenotypical analysis was performed on the corresponding knockout strains to determine the regulators' contribution to the (p)ppGpp-regulated stress response.

\section{Extracytoplasmic function sigma factor PF00052 or EcfG2}

$R$. etli PF00052 is the most highly upregulated alternative sigma factor during stationary phase in the wild type and upregulated over two-fold compared to the rsh mutant. Analysis of the PF00052 knockout mutant showed decreased survival following oxidative stress, approximately three orders of magnitude lower compared to the wild type (Figure 7). In accordance, the rsh mutant also displays an increased oxidative stress phenotype [30]. In contrast to the rsh mutant, however, the PF00052 mutant does not show a decreased viability after osmotic or heat shock.

Recently, a comprehensive phylogenetic analysis classified the ECF sigma factor family into 43 groups supported by domain architecture, genomic context conservation and potential targets [47]. R. etli contains

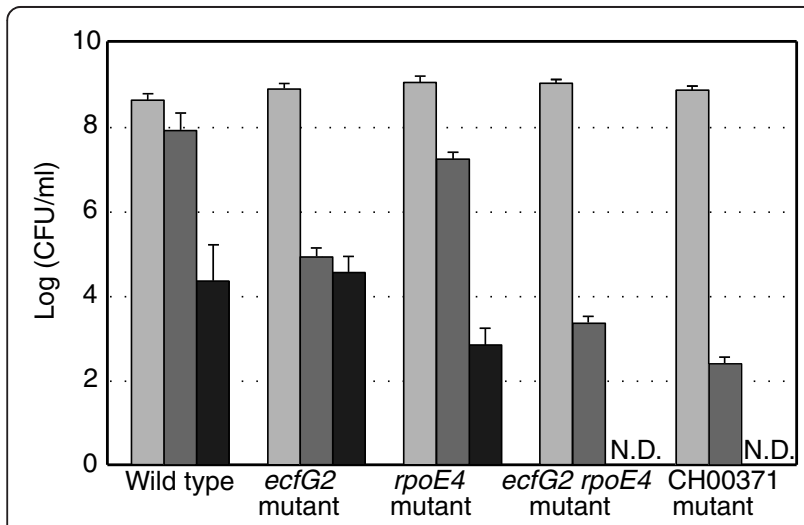

Figure 7 Stress survival of (p)ppGpp-dependent regulator mutants. Survival of the wild type, ecfG2 mutant, rpoE4 mutant, ecfG2-rpoE4 mutant and CH00371 mutant was determined by plating on TY medium after stress treatment and is shown as the mean log(colony forming units (CFU)/ml) of three biological replicates with error bars corresponding to standard deviations. Light gray bars represent control samples incubated at $30^{\circ} \mathrm{C}$ for the same time period as test samples. Dark gray bars represent samples incubated for 30 minutes in the presence of $10 \mathrm{mM} \mathrm{H}_{2} \mathrm{O}_{2}$ at $30^{\circ} \mathrm{C}$. Black bars represent samples incubated for 30 minutes at $45^{\circ} \mathrm{C}$. N.D., no colonies detected.
18 ECF sigma factors, of which three (rpoE4, sigK and PE00004), in addition to PF00052, were expressed during growth arrest as well. RpoE4 and PF00052 are 42\% identical and both belong to theECF15/EcfG group, which exclusively contains $\alpha$-proteobacterial ECFs, such as EcfG1 of Methylobacterium extorquens, RpoE2 of $S$. meliloti and SigT of C. crescentus. This group of proposed general stress response sigma factors is characterized by a conserved genomic context that encodes an EcfG-like sigma factor, a cytoplasmic NepR-like antisigma factor, a PhyR-like response regulator and a sensor histidine kinase. When the latter perceives a signal, it phosphorylates the regulator, which in turn binds to the anti-sigma factor, thereby releasing the sigma factor and initiating a signal transduction cascade [47]. In case of $R$. etli rpoE4, an anti-sigma factor $(\mathrm{CH} 03274)$ and a

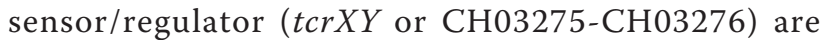
found upstream of rpoE4, and $\operatorname{tcr} X$ is transcribed in a strongly (p)ppGpp-dependent way (Additional file 3). Though PF00052 is also a member of the EcfG group, a similar genomic context was not recognized. Several other $\alpha$-proteobacteria also have two EcfG representatives, one that is present in the conserved genomic context while the other is not - for example, S. meliloti 1021 and Agrobacterium tumefaciens str. C58 [47]. A revised ECF nomenclature has been proposed [47] and has recently been adopted for EcfG-like ECF sigma factors in M. extorquens [43] and Bradyrhizobium japonicum [75]. Accordingly, we will hereafter refer to PF00052 as EcfG2.

Due to the high similarity between RpoE4 and EcfG2, we also analyzed the stress sensitivity of an rpoE4 mutant. This revealed a decrease in survival of one order of magnitude upon oxidative and heat stress compared to the wild type (Figure 7). Hence, the rpoE4 mutant exhibits an oxidative stress phenotype less severe than the ecfG2 mutant but, in contrast to the latter, a significant heat stress phenotype. This suggests that RpoE4 and EcfG2 control expression of, at least in part, non-overlapping sets of target genes. To determine if both sigma factors function completely independently in these stress responses, an rpoE4-ecfG2 double mutant was constructed. After heat or oxidative stress treatment, the double mutant showed higher sensitivity than either of the single mutants separately (Figure 7). Moreover, survival of the double mutant is even lower than expected based upon the respective phenotypes of the single mutants, indicating a synergistic effect. Hence, we conclude that both sigma factors have partially overlapping functions in the (p)ppGpp-mediated stress response of $R$. etli to the tested stress conditions.

$R$. etli has two heat shock sigma factors that may contribute to the observed heat stress phenotype. RpoH1 was shown to be the main heat shock sigma factor, 
though a more complete response requires $\mathrm{RpoH} 2$ [76]. The promoter region of both sigma factors contains an EcfG-like binding site.

Recently, expression of $R$. etli rpoH 2 was shown to be under positive control of RpoE4, while expression of rpoH1 is not [42]. To determine whether either or both rpoH sigma factors are regulated by EcfG2, their expression was analyzed by qPCR. The expression level of rpoH1 was not altered in the ecfG2 mutant compared to the wild type, while the transcription level of $r p o H 2$ was reduced by approximately $25 \%$. In the rpoE4-ecfG 2 double mutant, expression of $r p o H 2$ was reduced over 100fold, confirming its RpoE4-dependency (data not shown). In contrast, the level of rpoH1 was upregulated over 2.5fold in the double mutant. This increase is more likely a way to compensate for the impaired heat shock response, rather than a negative control of its expression.

Partial redundancy of EcfG-like sigma factors may occur in other $\alpha$-proteobacteria as well. In S. meliloti, two EcfG-like proteins are encoded by rpoE2 and rpoE5. RpoE2 was previously reported to regulate many stress response genes during stationary phase. In agreement with our observations, an S. meliloti rpoE2 mutant showed increased sensitivity to $\mathrm{H}_{2} \mathrm{O}_{2}$ during stationary phase and rpoE5 is upregulated under heat stress $[77,78]$.

\section{Putative transcriptional regulator $\mathrm{CH} 00371$ or PhrR}

$\mathrm{CH} 00371$ encodes a putative DNA-binding transcriptional regulator of unknown function belonging to the xenobiotic response element family. Expression of this gene is under positive (p)ppGpp-control during all growth phases, although most pronounced upon growth arrest. $\mathrm{CH} 00371$ is $80 \%$ identical to $\mathrm{PhrR}$, a putative repressor protein of $S$. meliloti. This regulator was shown to be induced by low $\mathrm{pH}$, hence designated $\mathrm{PhrR}$ for $\mathrm{pH}$-regulated [79]. In order to investigate whether $\mathrm{CH} 00371$ plays a role in the acid stress response, growth of the $R$. etli wild type and CH00371 mutant was examined at $\mathrm{pH}$ levels ranging from $\mathrm{pH} 3.5$ to 10 . No growth difference was observed, either at acidic or basic $\mathrm{pH}$ (data not shown). In addition to low $\mathrm{pH}$, oxidative stress agents and heat shock at neutral $\mathrm{pH}$ induce $p h r R$ in $S$. meliloti as well. Therefore, survival of the CH00371 mutant was determined under oxidative stress and after heat shock. Compared to the wild type, survival decreased by over four orders of magnitude upon oxidative shock following exposure to hydrogen peroxide (Figure 7). No survival was observed after heat treatment. Moreover, a plate assay demonstrated growth inhibition of a $\mathrm{CH} 00371$ mutant on medium containing $\mathrm{H}_{2} \mathrm{O}_{2}$ but not in the presence of the superoxide generators menadione and paraquat, nor of the organic hydroperoxide producer cumene hydroperoxide (data not shown).
In order to identify downstream elements in the regulatory cascade mediated by $\mathrm{CH} 00371$ during $R$. etli growth arrest, we carried out qPCR expression analysis of a selection of genes presumed to be associated with the oxidative and heat stress responses (Additional file 6). These candidate target genes were selected based on a literature search and sequence analysis. Several of these genes were downregulated. $\operatorname{rec} A$, a key regulator of the SOS response involved in DNA repair, and osmC, an osmotically induced peroxidase, were $70 \%$ and $30 \%$ downregulated in the $\mathrm{CH} 00371$ mutant compared to the wild type, respectively. The superoxide dismutase $\operatorname{sod} C$ was downregulated by $20 \%$. Surprisingly, expression of $k a t G$ was not significantly altered despite the oxidative stress phenotype. However, the expression level of katG was very low. This may indicate that KatG primarily exerts its function upon induction by oxidative shock. In addition, oxidative homeostasis is likely impaired in the $\mathrm{CH} 00371$ mutant as 14 genes related to oxidative stress resistance, such as $g s h B$, sufBCD and cysK, showed increased expression of over $25 \%$ compared to the wild type. Interestingly, four genes of the EcfG2-RpoE4-regulon (CH00600, $\mathrm{CH} 01778, \mathrm{CH} 01802$ and $\mathrm{CH} 02172)$ were also downregulated over $25 \%$, possibly contributing to the observed heat stress phenotype.

Furthermore, the expression level of $\mathrm{CH} 00371$ was not altered in the ecfG2 mutant and ecfG2 rpoE4 double mutant compared to the wild type, nor vice versa (data not shown). Hence, these novel regulators exert their role in the observed stress phenotypes of the respective mutants independently of each other.

\section{Putative serine kinase CH02817 or PrkA}

$\mathrm{CH} 02817$ or prkA is upregulated over 27 -fold in the wild type compared to the rsh mutant during stationary phase, making it the most strongly (p)ppGpp-induced gene detected in our array. This was confirmed by RTqPCR (Figure 3). PrkA belongs to the PrkA family of serine protein kinases and is highly conserved, with homologs in many eubacteria and archae suggesting a conserved function. E. coli and B. subtilis PrkAs were shown to phosphorylate serine residues of proteins $[80,81]$ and display $66 \%$ and $34 \%$ identity with the $R$. etli ortholog, respectively. Protein phosphorylation usually changes the function of the target by modulating its activity, its localization or interaction with other proteins, thereby converting extracellular signals into cellular responses, such as adaptation of the central metabolism, production of secondary metabolites and pathogenicity [82]. Although the specific regulatory function of PrkA remains unknown, the $B$. subtilis ortholog was shown to be an important inner spore coat protein under control of the developmental sigma factor $\sigma^{\mathrm{E}}$ [83]. Furthermore, prkA is part of a highly conserved gene cluster together with the two downstream genes 
$\mathrm{CH} 02816$ and $\mathrm{CH} 02815$. This suggests that prkA is likely the first gene of a three-gene operon in $R$. etli (Figure $8 \mathrm{a}$ ), which we confirmed by RT-PCR (data not shown). The second operon gene encodes a protein containing a von Willebrand Factor type A domain and the third encodes a SpoVR-like protein. So far, these genes have not been functionally characterized.

To determine the function of prkA in $R$. etli, we constructed a non-polar deletion mutant. A phenotypical analysis of this mutant, including survival during stationary phase, osmotic stress, oxidative stress and heat stress, revealed no clear stress phenotype. Therefore, PrkA does not seem to play a crucial role in the stress response of $R$. etli. This is rather unexpected given the high level of (p)ppGpp-dependency and strong induction under stress conditions in other organisms. In E. coli and $S$. Typhimurium, the prkA homolog, annotated as yeaG, was also shown to be highly upregulated by (p) ppGpp upon entry into stationary phase as part of the RpoS regulon [81]. In addition, yea $G$ is upregulated by Lrp during stationary phase in E. coli $[45,46]$. Other
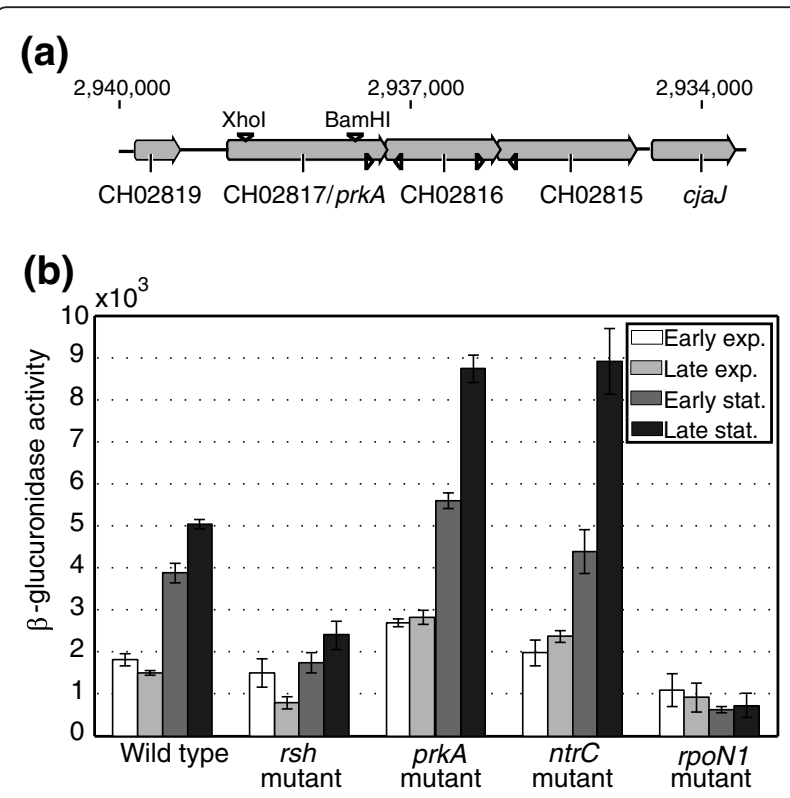

Figure 8 prkA genomic context and expression analysis. (a) $R$. etli prkA is the first gene of a three-gene operon. Open reading frames are represented by right facing arrows, genomic coordinates are indicated above. Restriction sites for the deletion insertion of the prkA mutant are depicted by downwards facing triangles, and primer sites for RT-PCR used to determine prkA operon structure are depicted by left and right facing triangles. (b) Expression of prkAgusA transcriptional reporter fusion was monitored in different $R$. etli mutant backgrounds during growth in AMS succinate. The strains were the wild type $R$. etli CFN42, rs mutant, prkA mutant, ntrC mutant and rpoN1 mutant. Expression levels are shown in Miller units and are the means of three biological replicates with error bars representing the standard deviation. Exp., exponential; Stat., stationary. stress conditions were also reported to induce yeaG expression, such as acid and osmotic stress in E. coli and sublethal concentrations of polymyxin in $S$. Typhimurium [6].

Additionally, PrkA was postulated to be involved in nitrogen metabolism or the nitrogen starvation response in $E$. coli based on its potential association with NtrCB and $G \ln P[46,84]$. To examine the transcriptional regulation of prkA in R. etli, we monitored expression of a transcriptional prkA-gusA promoter fusion in various genetic backgrounds (Figure $8 \mathrm{~b}$ ). $\beta$-Glucuronidase activity was measured during exponential and stationary phase under the same conditions used for microarray sampling, confirming that the expression of prkA is highly upregulated upon growth arrest in the wild type and positively controlled by (p)ppGpp. Moreover, increased expression of prkA was observed in a prkA mutant, indicating that $p r k A$ is negatively autoregulated. Expression of prkA was also shown to be regulated by $\mathrm{NtrC}$ and RpoN1. NtrC is a transcriptional regulator involved in nitrogen assimilation and growth in nitrogen-limited conditions, as well as a member of the $\sigma^{\mathrm{N}}$ dependent activator family [85]. RpoN1 codes for the main $\sigma^{\mathrm{N}}$ operating under free-living growth conditions in $R$. etli [86]. In the rpoN1 mutant background, prkA showed an even stronger downregulation than in the rsh mutant during stationary phase, showing prkA transcription to be strongly $\sigma^{\mathrm{N}}$-dependent. Because $\mathrm{NtrC}$ is a common activator of $\sigma^{\mathrm{N}}$-dependent genes, a similar downregulation of prkA in the $n t r C$ mutant was expected. However, no downregulation of $p r k A$ was observed during growth and early stationary phase in the $n t r C$ mutant. Instead, prkA was highly upregulated during late stationary phase compared to the wild type, suggesting that $p r k A$ is under negative control of $\mathrm{NtrC}$.

To further analyze the role of PrkA in cellular metabolism, we compared growth of the wild type and prkA mutant on 384 different nitrogen sources using glucose as the sole carbon source. Even though transcriptional control of prkA expression by rpoN1 suggests an involvement for PrkA in nitrogen metabolism, no growth defects were detected. Therefore, the specific function of this highly conserved protein in the (p)ppGpp regulon remains to be identified.

\section{Conclusions}

Analysis of growth phase-specific gene expression of the $R$. etli wild type and $r s h$ mutant has provided insight into the (p)ppGpp regulon of $R$. etli, providing the first genome-wide view of the stringent response in an $\alpha$ proteobacterium. Our results indicate that (p)ppGpp functions as a global regulator, with primarily an inducing role, in the adaptation to a non-growing lifestyle as shown by the extensive differential expression of genes 
belonging to all functional categories. Moreover, we showed both similarities and differences to its role in $E$. coli and other bacteria, reflecting the merit of investigating a well-studied regulatory response in species more distantly related to typical model organisms. Surprisingly, even though (p)ppGpp is considered to be a growtharrest specific messenger, we identified a significant number of (p)ppGpp-dependent genes during early exponential phase as well, suggesting functional relevance of the low basal level of (p)ppGpp during active growth in $R$. etli. Additionally, the genome-wide transcriptome analysis of a strain deficient in a global regulator, and exhibiting a pleiotropic phenotype, enabled us to identify diverse regulators that control genes associated with a subset of stress phenotypes. The phenotypic analysis of three novel downstream regulators during stationary phase, that is, ecfG2, CH00371, and prkA, allowed us to obtain additional insight into the intricate regulatory role of this stress alarmone (Figure 9). Added detail to the complex picture of (p)ppGpp-dependent regulation of gene expression in $R$. etli was further provided by identifying several up- and downstream elements in the signal transduction cascades of these regulators. We conclude that (p)ppGpp is situated high up in the hierarchy of cellular gene regulation of $R$. etli, orchestrating its adaptation to growth stage or extracellular conditions through specific downstream regulators to control expression of a variety of target genes.

\section{Materials and methods}

\section{Bacterial strains and growth conditions}

The bacterial strains and plasmids used for this work are listed in Additional file 7. R. etli CFN42 strains were cultured in minimal AMS or complex TY medium at $30^{\circ} \mathrm{C}$ when used for RNA isolation or stress tests, respectively $[29,87]$. AMS medium was supplemented with $10 \mathrm{mM} \mathrm{NH}_{4} \mathrm{Cl}$ and $10 \mathrm{mM}$ succinate unless otherwise indicated. E. coli strains were grown at $37^{\circ} \mathrm{C}$ in $\mathrm{LB}$ medium. In order to study gene expression during different growth phases in AMS medium, samples were taken based on optical density (OD) readings of $\mathrm{OD}_{600}$ $=0.3, \mathrm{OD}_{600}=0.7$, and 6 hours after reaching the

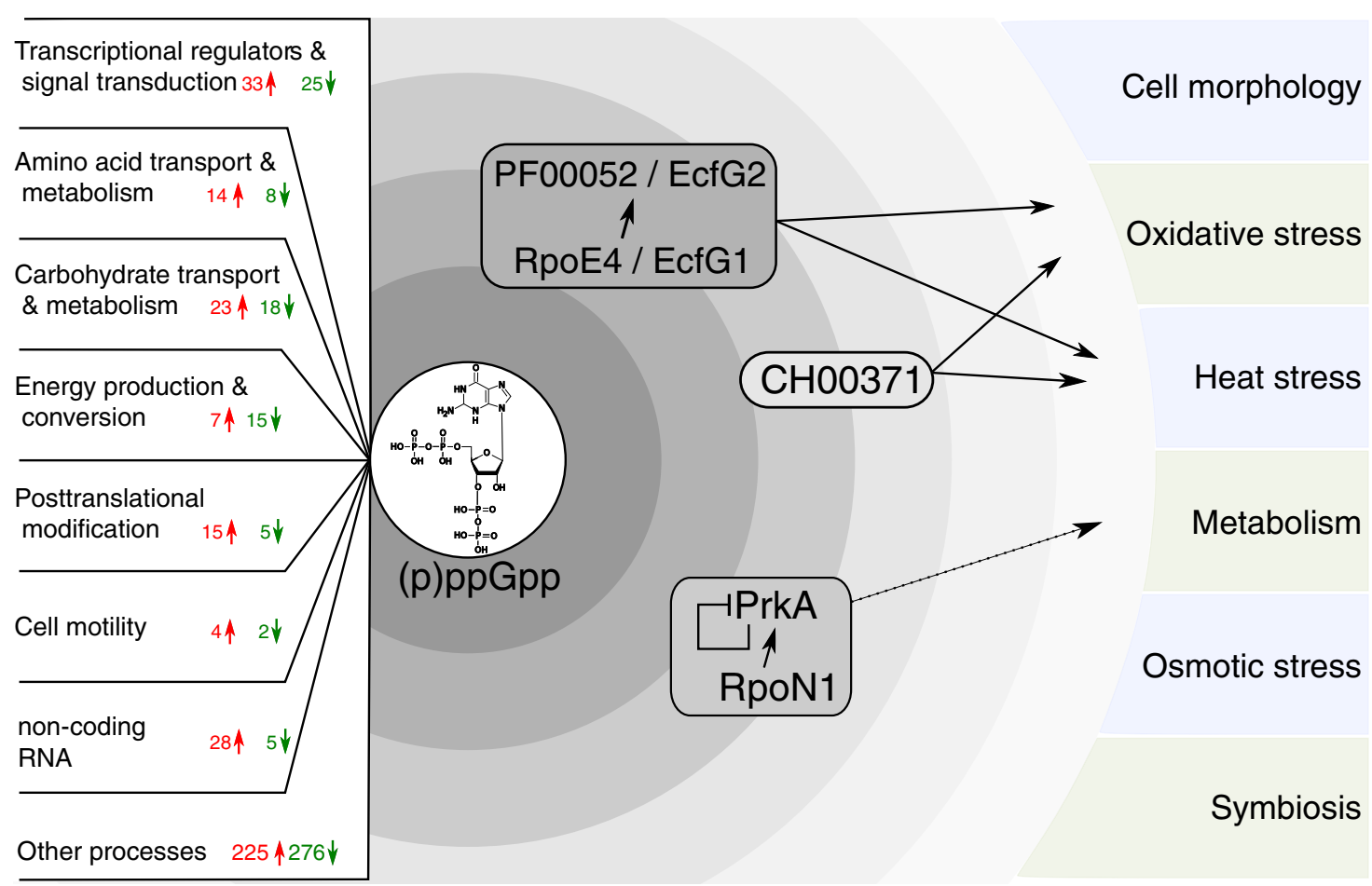

Figure 9 The (p)ppGpp regulon of $\boldsymbol{R}$. etli. The extensive impact of (p)ppGpp on gene expression of R. etli is illustrated by the number of upand downregulated genes grouped according to functional categories. The remaining categories are combined as 'Other processes'. The rsh mutant is unable to synthesize (p)ppGpp and has a pleiotropic phenotype, such as an altered morphology, increased stress sensitivity and impaired symbiosis. As a global regulator, the regulon of (p)ppGpp is multilayered. Further insight into the (p)ppGpp-dependent stress response was obtained by the identification and subsequent characterization of three different regulators that are under strong positive regulation of ( $p$ ) ppGpp during stationary phase. EcfG2/PF00052 and RpoE4, both ECF sigma factors, are partly functionally redundant for survival under heat stress and oxidative stress. The transcription factor $\mathrm{CH} 00371$ is also involved in survival during both heat and oxidative stress. PrkA, a serine kinase, likely plays a role in the (p)ppGpp-dependent adaptation of the cellular metabolism. Its transcription is positively controlled by RpoN1 and negatively autoregulated. 
maximum optical density $(\mathrm{OD})_{600}$, representing early exponential, late exponential and stationary phase, respectively [32]. Antibiotics were supplied at the following final concentrations (in $\mu \mathrm{g}^{-1} \mathrm{ml}$ ): ampicillin, 100; gentamicin, 50; kanamycin, 25; spectinomycin, 50; nalidixic acid, 15; neomycin, 60; and tetracycline, 1 (E. coli) or 0.1 (R. etli).

\section{Mutant construction}

The R. etli CFN42 rsh mutant was constructed by insertion of an $\mathrm{Sp}^{\mathrm{R}}$ cassette, obtained from pHP $45 \Omega \mathrm{Sp}$, as described previously for $R$. etli CNPAF512 [29]. The ecfG2 and $\mathrm{CH} 00371$ mutants were constructed by first amplifying a $2.5-\mathrm{kb}$ and a $1.9-\mathrm{kb}$ fragment, respectively, using $P f x$ DNA polymerase and primers (CACCG CGGCCGCGGGTTT AAGGGGATAAATT and ACT GGCGGCCGCAAG GGCCGATCGAGATCCAC in the case of ecfG2; CACCGCGGCCGCAGCTGC AGGATCT TATGGGAATA and ACTGGCGGCCGCCGACGACCAGATCCTGAT CGC in the case of CH00371) that carried NotI recognition sites at their 5 ' ends (shown in italics). These fragments were subsequently cloned into pUC18Not, and a $\mathrm{Km}^{\mathrm{R}}$ cassette flanked by transcription termination signals, obtained from pHP $45 \Omega \mathrm{Km}$, was inserted in the HindIII and EcoRV site of CH00371 and ecfG2, respectively. From these plasmids, the corresponding NotI fragments were cloned into the suicide plasmid pJQ200uc1. For construction of the rpoE4ecfG2 double mutant, an ecfG2:: $\Omega$ Sp suicide construct was obtained as described for the ecfG2 mutant above, replacing the $\mathrm{Km}^{\mathrm{R}}$ cassette with a $\mathrm{Sp}^{\mathrm{R}}$ cassette.

The non-polar prkA mutant was constructed by amplifying a 3.6-kb fragment using $P f x$ DNA polymerase and primers (CACCGTTAACTCGACAGGAAAAGGTAG AGC and CACCGTTAACTACTCG TCAAGAAGGAGGCT) that carried HpaI recognition sites at their 5' ends (shown in italics). This fragment was cloned into pCR4Blunt-TOPO (Invitrogen, Carlsbad, CA, USA). A fragment of $1.2 \mathrm{~kb}$ was removed from prkA by digesting with BamHI and XhoI (Figure 8a) and the construct was ligated after blunting, creating a deletion in prkA. An HpaI fragment was removed form this construct and cloned into the SmaI site of PJQ200uc1.

Finally, these suicide constructs were used for sitedirected mutagenesis of the respective genes following triparental conjugation as described previously [88]. The obtained mutants were verified by Southern blot hybridization.

\section{RNA isolation and CDNA synthesis for microarray detection}

RNA was isolated as described previously [32]. Briefly, the RNA content of bacterial cultures was stabilized using a phenol:ethanol solution. Pellets were frozen in liquid nitrogen and stored at $-80^{\circ} \mathrm{C}$. Total RNA was extracted using the TRIzol Plus RNA Purification kit (Invitrogen). DNA contamination was removed by TURBO DNase (Ambion, Austin, TX, USA)) and afterwards checked by PCR ( 45 cycles). To increase RNA yields and account for experimental variation, RNA from six different cultures was pooled. RNA integrity was analyzed using Experion RNA StdSens Chips (Biorad, Hercules, CA, USA) before and after precipitation. All samples had an RNA Quality Indicator value of 10. RNA quantity and purity was assessed using the NanoDrop ND-1000. The A260/A280 ratio and A260/ A230 ratio of all samples were $\geq 2$.

cDNA was synthesized using random decamers (Ambion) and the SuperScript Double-Stranded cDNA Synthesis Kit (Invitrogen) according to the manufacturer's protocol.

\section{High-density microarray design and data preprocessing}

A whole-genome tiling array covering the entire $R$. etli genome sequence was used (see GEO GPL9409) and the data were analyzed as described previously [32]. Samples were hybridized and scanned by NimbleGen. The data were deposited in the NCBI Gene Expression Omnibus (GEO) and can be accessed through accession numbers [GEO: GSE23961], [GEO:GSM462173], [GEO:GSM462178], [GEO:GSM462180], [GEO:GSM590285], [GEO:GSM 590286] and [GEO:GSM590287].

Differentially expressed genes were identified based on a standard deviation cutoff. These genes were considered induced or repressed if the absolute expression ratio was $\geq 2\left(\log _{2} \geq 1\right)$. This threshold is cogent since most regulatory responses in nature appear to function using low level changes as a kind of energy saving solution [89]. Hierarchical clustering was performed using the software package $\mathrm{R}$.

\section{RT-(q)PCR}

Expression levels were determined by RT-qPCR using SYBR Green, as described previously [32]. In short, primers were designed using Primer Express 3.0. Pooled total RNA $(2 \mu \mathrm{g})$ of each growth condition (early/late exponential phase, stationary phase) was reverse transcribed to single-stranded cDNA using the SuperScript VILO cDNA Synthesis Kit according to the manufacturer's instructions (Invitrogen). DNA contamination of the RNA samples was checked by PCR ( 45 cycles) before RT. cDNA (40 ng) was used in each reaction. All reactions were performed in triplicate.

The microarray data were validated by determining the expression levels of 14 representative genes: flaCh1, potF, $r p s H, f l g B, r p l R, o t s A, a g l E$, a serine tRNA (CH01348), and genes encoding a chaperonin GroEL (CH00828), the sigma 54 modulation protein $(\mathrm{CH} 00406)$, a permease 
protein of the Nod factor $A B C$ transporter family (PD00277), the serine protein kinase prkA/CH02817, the transcriptional regulator $\mathrm{CH} 00371$ and the ECF sigma factor ecfG2/PF00052. The $\log _{2}$ ratios of the array data were compared to the $\log _{2}$ ratios of the qPCR results. $16 \mathrm{~S}$ rRNA was not used as a reference gene as the level of mRNA/rRNA fluctuates during growth and the expression of rRNA is controlled by (p)ppGpp. New reference genes were identified using the geNorm algorithm in order to normalize the qPCR data [90]. Based on the microarray data, five genes were chosen that were relatively stable across all samples and assumed not to be co-regulated. These candidate reference genes were greA, $\operatorname{cin} R a, \operatorname{tat} A$, and genes encoding a zinc binding protein (CH00586) and a hypothetical protein (CH01579). 16S rRNA was included for comparison. Using geNorm, we determined repBa2 and tat $A$ to be the most stable reference genes as they have the lowest gene expression stability values M (Figure S3a in Additional file 8). Consequently, a gene expression normalization factor could be calculated for each sample using the most stable genes. By plotting the pairwise variation $\mathrm{V}$ between two sequential normalization factors containing an increasing number of genes, we determined that the three best reference genes were an optimal number of reference genes for normalization (Figure S3b in Additional file 8). Although $V_{2 / 3}$, the pairwise variation between the normalization factors calculated by the two and three most stable genes, is strictly higher then the proposed 0.15 cutoff value of Vandesompele et al. [90], the difference is very small and the pairwise variation decreases only slightly by taking an additional fourth reference gene. Therefore, the normalization factor would not significantly change if more internal control genes were to be included. Also, the degree of resolution does not require a fourth reference gene.

RT-PCR was performed on cDNA samples (40 ng) of stationary phase to determine the operon organization of $p r k A$. The primers were designed accordingly (Additional file 6). Taq DNA polymerase was used in the PCR reactions (35 cycles).

\section{Construction of prkA-gusA promoter fusion and $\beta$ - glucuronidase assay}

The prkA-gusA reporter fusion was constructed by first amplifying the $400 \mathrm{bp}$ upstream of prkA by PCR using Pfx DNA polymerase and primers (ACTG AAGCTTT CTGCGGTTCGCCTATCGCA and ACTGTCTAGA AGCGCCGGAAG CGTATGATC) that carried a HindIII and XbaI recognition site at their 5' end (shown in italics), respectively. This promoter fragment was cloned into pFAJ1703 after digestion with HindIII and XbaI, thereby flanking the promoterless 5' end of gusA. Quantitative analysis of GusA activity was carried out as described previously [29].

\section{Stress and stationary phase survival}

To study stress survival, wild-type and mutant cells from a freshly grown culture on a MM79 agar plate were resuspended in $10 \mathrm{mM} \mathrm{MgSO}_{4}$ at an $\mathrm{OD}_{600}$ of approximately 0.5 . For each regulator, two independently constructed mutants were analyzed in order to exclude the involvement of secondary mutations. To test heat stress survival, $1 \mathrm{ml}$ of each sample was incubated at $45^{\circ} \mathrm{C}$ for 30 minutes. In case of oxidative stress, $0.1 \mathrm{ml}$ of 100 $\mathrm{mM} \mathrm{H}_{2} \mathrm{O}_{2}$ was added to $0.9 \mathrm{ml}$ of each sample for 30 minutes or 1 hour while for osmotic stress $0.5 \mathrm{ml}$ of 5 $\mathrm{M} \mathrm{NaCl}$ was added to $0.5 \mathrm{ml}$ of sample. Samples were plated on TY agar containing nalidixic acid using the Eddy Jet spiral plater (IUL Instruments, Barcelona, Spain). Control samples were incubated without the stress agent at $30^{\circ} \mathrm{C}$ and the colony forming units (CFU) were determined at the same time point as the stressed samples. The total number of CFU per $\mathrm{ml}$ was determined after 3 days of incubation at $30^{\circ} \mathrm{C}$ using the Flash and Go automated colony counter (IUL Instruments). All experiments were repeated at least two times using three independent biological replicates.

To assess long-term survival, pellets of overnight cultures of wild-type and mutant strains were washed and resuspended in $10 \mathrm{mM} \mathrm{MgSO}_{4}$ at an $\mathrm{OD}_{600}$ of 0.5 . A volume of $100 \mathrm{ml}$ of AMS medium $\left(10 \mathrm{mM} \mathrm{NH}_{4} \mathrm{Cl}\right.$ and succinate) was inoculated with $1 \mathrm{ml}$ of cell suspension and incubated at $30^{\circ} \mathrm{C}$ for 2 weeks. Samples of $1 \mathrm{ml}$ were removed at the indicated time points and subjected to viable cell counts as described above.

\section{Swimming test}

To study swimming activity, TY plates containing $0.2 \%$ agar were spot inoculated with cultures in exponential phase and incubated at $30^{\circ} \mathrm{C}$ in a closed container as described previously [91]. Each strain was tested fivefold in two independent experiments. The swimming halo diameter was measured after one, two and three days.

\section{Growth analysis}

Biolog Phenotype Microarray panels PM3/6/7/8 were used to test growth on nitrogen sources and PM10 was used to test $\mathrm{pH}$ susceptibility (Biolog, Hayward, CA, USA). AMS medium was inoculated $(1: 1,000)$ with overnight cultures of $R$. etli strains, washed and the $\mathrm{OD}_{600}$ was corrected to approximately 0.5 . No $\mathrm{NH}_{4} \mathrm{Cl}$ was added in case of PM3/6/7/8. The Biolog redox indicator dye Mix A was added to the medium (1:100). The microplates were loaded with $100 \mu \mathrm{l}$ in each well and incubated for 7 days at $30^{\circ} \mathrm{C}$. Dye reduction was monitored every $12 \mathrm{~h}$ by measuring the $\mathrm{OD}_{570}$ using a Synergy Mx Microplate Reader (BioTek, Winooski, VT, USA). 


\section{Additional material}

Additional file 1: Figure S1. Growth curve of R. etli CFN42 in AMS medium. (a) Optical density (OD) readings during growth of the wild type and rsh mutant shown in green and red, respectively. The arrows indicate the time points of sampling. (b) Colony forming units (CFU) during growth of the wild type and rsh mutant.

Additional file 2: Figure S2. MA plots comparing transcriptome data. Scatter plots of the microarray data that plot the distribution of the $\log _{2}$ intensity ratio (M-value) versus the $\log _{2}$ average intensity (A-value). Differentially expressed genes that are upregulated or downregulated are shown in red or green, respectively. The number of genes with a growth phase or (p)ppGpp-dependent expression profile are indicated by histogram bars at the right of the MA plot. (a) Wild type compared to rsh mutant in stationary phase. (b) Wild type compared to rsh mutant in exponential phase.

Additional file 3: Table S1. The differentially expressed genes during stationary phase and exponential phase in the wild type compared to the rsh mutant.

Additional file 4: Table S2. The RpoE4-regulated genes according to Martinez-Salazar et al. (2009) that were found to be alarmone-dependent in this study [42].

Additional file 5: Table S3. The alarmone-dependent ncRNAs.

Additional file 6: Table S4. The RT-qPCR fold changes compared to array fold changes and $\mathrm{QPCR}$ primers.

Additional file 7: Table S5. The bacterial strains and plasmids used in this study.

Additional file 8: Figure S3. RT-qPCR identification of stable endogenous genes. (a) Determining the most stable reference genes using the average expression stability value $\mathrm{M}$ of the remaining reference genes during a stepwise exclusion of the least stable internal control gene. The genes are ranked according to increasing expression stability. At the left are the least stable genes and at the right are the most stable ones. (b) Determining the optimal number of reference genes using the pairwise variation $V$ between two sequential normalization factors containing an increasing number of genes with 0.15 as a proposed cutoff value by Vandesompele et al. [90].

\section{Abbreviations}

CFU: colony forming units; ECF: extracytoplasmic function; IS: insertion sequence; ncRNA: non-coding RNA; OD: optical density; ppGpp: guanosine tetraphosphate; pppGpp: guanosine pentaphosphate; RNAP: RNA polymerase; $r$ rn: ribosomal RNA; RT-qPCR: reverse transcription-quantitative polymerase chain reaction.

\section{Acknowledgements}

$\mathrm{MV}$ is indebted to the Institute for the Promotion of Innovation through Science and Technology in Flanders (IWT-Flanders). This work was supported by grants from the Research Council of the KU Leuven (GOA/011/2008) and from the Fund for Scientific Research-Flanders (G.0637.06 and G.0412.10).

\section{Authors' contributions}

$\mathrm{MV}$ carried out the experiments and bioinformatics analysis. MV, MF, KB, and $J M$ conceived the study and contributed to the interpretation of the data. $L C, K E$, and $K M$ performed and contributed to the microarray data normalization and processing. MV, MF and JM were involved in drafting the manuscript. All authors read and approved the final manuscript.

Received: 19 November 2010 Revised: 1 February 2011 Accepted: 16 February 2011 Published: 16 February 2011

\section{References}

1. Fauvart M, Michiels J: Rhizobial secreted proteins as determinants of host specificity in the rhizobium-legume symbiosis. FEMS Microbiol Lett 2008, 285:1-9.
2. Gibson KE, Kobayashi H, Walker GC: Molecular determinants of a symbiotic chronic infection. Annu Rev Genet 2008, 42:413-441.

3. Masson-Boivin C, Giraud E, Perret X, Batut J: Establishing nitrogen-fixing symbiosis with legumes: how many rhizobium recipes? Trends Microbiol 2009, 17:458-466.

4. Downie JA: The roles of extracellular proteins, polysaccharides and signals in the interactions of rhizobia with legume roots. FEMS Microbiol Rev 2010, 34:150-170

5. Navarro Llorens JM, Tormo A, Martinez-Garcia E: Stationary phase in gramnegative bacteria. FEMS Microbiol Rev 2010, 34:476-495.

6. Tani TH, Khodursky A, Blumenthal RM, Brown PO, Matthews RG: Adaptation to famine: a family of stationary-phase genes revealed by microarray analysis. Proc Natl Acad Sci USA 2002, 99:13471-13476.

7. Potrykus K, Cashel M: (p)ppGpp: Still Magical? (*). Annu Rev Microbiol 2008, 62:35-51.

8. Chang DE, Smalley DJ, Conway T: Gene expression profiling of Escherichia coli growth transitions: an expanded stringent response model. Mol Microbiol 2002, 45:289-306.

9. Traxler MF, Chang DE, Conway T: Guanosine 3',5'-bispyrophosphate coordinates global gene expression during glucose-lactose diauxie in Escherichia coli. Proc Natl Acad Sci USA 2006, 103:2374-2379.

10. Braeken K, Moris M, Daniels R, Vanderleyden J, Michiels J: New horizons for (p)ppGpp in bacterial and plant physiology. Trends Microbiol 2006, 14:45-54.

11. Wolz C, Geiger T, Goerke C: The synthesis and function of the alarmone (p)ppGpp in firmicutes. Int J Med Microbiol 2010, 300:142-147.

12. Magnusson LU, Farewell A, Nystrom T: ppGpp: a global regulator in Escherichia coli. Trends Microbiol 2005, 13:236-242.

13. Srivatsan A, Wang JD: Control of bacterial transcription, translation and replication by (p)ppGpp. Curr Opin Microbiol 2008, 11:100-105.

14. Barker MM, Gaal T, Josaitis CA, Gourse RL: Mechanism of regulation of transcription initiation by ppGpp. I. Effects of ppGpp on transcription initiation in vivo and in vitro. J Mol Biol 2001, 305:673-688.

15. Gummesson B, Magnusson LU, Lovmar M, Kvint K, Persson O, Ballesteros M, Farewell A, Nystrom T: Increased RNA polymerase availability directs resources towards growth at the expense of maintenance. EMBO J 2009, 28:2209-2219.

16. Jishage M, Kvint K, Shingler V, Nystrom T: Regulation of sigma factor competition by the alarmone ppGpp. Genes Dev 2002, 16:1260-1270

17. Bernardo LM, Johansson LU, Solera D, Skarfstad E, Shingler V: The guanosine tetraphosphate (ppGpp) alarmone, DksA and promoter affinity for RNA polymerase in regulation of sigma-dependent transcription. Mol Microbiol 2006, 60:749-764.

18. Durfee T, Hansen AM, Zhi H, Blattner FR, Jin DJ: Transcription profiling of the stringent response in Escherichia coli. J Bacteriol 2008, 190:1084-1096.

19. Aberg A, Fernandez-Vazquez J, Cabrer-Panes JD, Sanchez A, Balsalobre C: Similar and divergent effects of ppGpp and DksA deficiencies on transcription in Escherichia coli. J Bacteriol 2009, 191:3226-3236.

20. Dalebroux ZD, Yagi BF, Sahr T, Buchrieser C, Swanson MS: Distinct roles of ppGpp and DksA in Legionella pneumophila differentiation. Mol Microbiol 2010, 76:200-219

21. Dean RE, Ireland PM, Jordan JE, Titball RW, Oyston PC: RelA regulates virulence and intracellular survival of Francisella novicida. Microbiology 2009, 155:4104-4113.

22. Yan X, Zhao C, Budin-Verneuil A, Hartke A, Rince A, Gilmore MS, Auffray Y, Pichereau V: The (p)ppGpp synthetase RelA contributes to stress adaptation and virulence in Enterococcus faecalis V583. Microbiology 2009, 155:3226-3237.

23. Kazmierczak KM, Wayne KJ, Rechtsteiner A, Winkler ME: Roles of rel in stringent response, global regulation and virulence of serotype 2 Streptococcus pneumoniae D39. Mol Microbiol 2009, 72:590-611.

24. Dalebroux ZD, Svensson SL, Gaynor EC, Swanson MS: ppGpp conjures bacterial virulence. Microbiol Mol Biol Rev 2010, 74:171-199.

25. Aviv M, Giladi H, Schreiber G, Oppenheim AB, Glaser G: Expression of the genes coding for the Escherichia coli integration host factor are controlled by growth phase, rpoS, ppGpp and by autoregulation. Mol Microbiol 1994, 14:1021-1031.

26. Lemke JJ, Durfee T, Gourse RL: DksA and ppGpp directly regulate transcription of the Escherichia coli flagellar cascade. Mol Microbiol 2009, 74:1368-1379. 
27. Wells DH, Long SR: The Sinorhizobium meliloti stringent response affects multiple aspects of symbiosis. Mol Microbiol 2002, 43:1115-1127.

28. Calderon-Flores A, Du Pont G, Huerta-Saquero A, Merchant-Larios H, ServinGonzalez L, Duran S: The stringent response is required for amino acid and nitrate utilization, nod factor regulation, nodulation, and nitrogen fixation in Rhizobium etli. J Bacteriol 2005, 187:5075-5083.

29. Moris M, Braeken K, Schoeters E, Verreth C, Beullens S, Vanderleyden J, Michiels J: Effective symbiosis between Rhizobium etli and Phaseolus vulgaris requires the alarmone ppGpp. J Bacteriol 2005, 187:5460-5469.

30. Braeken K, Fauvart M, Vercruysse M, Beullens S, Lambrichts I, Michiels J: Pleiotropic effects of a rel mutation on stress survival of Rhizobium etli CNPAF512. BMC Microbiol 2008, 8:219.

31. Gonzalez V, Santamaria RI, Bustos P, Hernandez-Gonzalez I, Medrano-Soto A, Moreno-Hagelsieb G, Janga SC, Ramirez MA, Jimenez-Jacinto V, ColladoVides J, Davila G: The partitioned Rhizobium etli genome: genetic and metabolic redundancy in seven interacting replicons. Proc Natl Acad Sci USA 2006, 103:3834-3839.

32. Vercruysse M, Fauvart M, Cloots L, Engelen $K$, Thijs IM, Marchal K, Michiels J: Genome-wide detection of predicted non-coding RNAs in Rhizobium etli expressed during free-living and host-associated growth using a highresolution tiling array. BMC Genomics 2010, 11:53.

33. Traxler MF, Summers SM, Nguyen HT, Zacharia VM, Hightower GA, Smith JT, Conway T: The global, ppGpp-mediated stringent response to amino acid starvation in Escherichia coli. Mol Microbiol 2008, 68:1128-1148.

34. Brockmann-Gretza O, Kalinowski J: Global gene expression during stringent response in Corynebacterium glutamicum in presence and absence of the rel gene encoding (p)ppGpp synthase. BMC Genomics 2006, 7:230.

35. Hesketh A, Chen WJ, Ryding J, Chang S, Bibb M: The global role of ppGpp synthesis in morphological differentiation and antibiotic production in Streptomyces coelicolor A3(2). Genome Biol 2007, 8:R161.

36. Martinez-Antonio A, Collado-Vides J: Identifying global regulators in transcriptional regulatory networks in bacteria. Curr Opin Microbiol 2003, 6:482-489

37. Todd JD, Sawers G, Rodionov DA, Johnston AW: The Rhizobium leguminosarum regulator IrrA affects the transcription of a wide range of genes in response to Fe availability. Mol Genet Genomics 2006, 275:564-577.

38. Singleton C, White GF, Todd JD, Marritt SJ, Cheesman MR, Johnston AW, Le Brun NE: Heme-responsive DNA binding by the global iron regulator Irr from Rhizobium leguminosarum. J Biol Chem 2010, 285:16023-16031.

39. Vinella D, Albrecht C, Cashel M, D'Ari R: Iron limitation induces SpoTdependent accumulation of ppGpp in Escherichia coli. Mol Microbiol 2005, 56:958-970.

40. Miethke M, Westers $\mathrm{H}$, Blom EJ, Kuipers OP, Marahiel MA: Iron starvation triggers the stringent response and induces amino acid biosynthesis for bacillibactin production in Bacillus subtilis. J Bacteriol 2006, 188:8655-8657.

41. Dombrecht B, Heusdens C, Beullens S, Verreth C, Mulkers E, Proost P, Vanderleyden J, Michiels J: Defence of Rhizobium etli bacteroids against oxidative stress involves a complexly regulated atypical 2-Cys peroxiredoxin. Mol Microbiol 2005, 55:1207-1221.

42. Martinez-Salazar JM, Salazar E, Encarnacion S, Ramirez-Romero MA, Rivera J: Role of the extracytoplasmic function sigma factor RpoE4 in oxidative and osmotic stress responses in Rhizobium etli. J Bacteriol 2009, 191:4122-4132.

43. Francez-Charlot A, Frunzke J, Reichen C, Ebneter JZ, Gourion B, Vorholt JA: Sigma factor mimicry involved in regulation of general stress response. Proc Natl Acad Sci USA 2009, 106:3467-3472.

44. Balleza E, Lopez-Bojorquez LN, Martinez-Antonio A, Resendis-Antonio O, Lozada-Chavez I, Balderas-Martinez YI, Encarnacion S, Collado-Vides J: Regulation by transcription factors in bacteria: beyond description. FEMS Microbiol Rev 2009, 33:133-151.

45. Ibanez-Ruiz M, Robbe-Saule V, Hermant D, Labrude S, Norel F: Identification of RpoS (sigma(S))-regulated genes in Salmonella enterica serovar typhimurium. J Bacterio/ 2000, 182:5749-5756.

46. Weber H, Polen T, Heuveling J, Wendisch VF, Hengge R: Genome-wide analysis of the general stress response network in Escherichia coli: sigmaS-dependent genes, promoters, and sigma factor selectivity. $J$ Bacteriol 2005, 187:1591-1603.

47. Staron A, Sofia HJ, Dietrich S, Ulrich LE, Liesegang H, Mascher T: The third pillar of bacterial signal transduction: classification of the extracytoplasmic function (ECF) sigma factor protein family. Mol Microbiol 2009, 74:557-581.

48. Kanehara K, Ito K, Akiyama Y: YaeL (EcfE) activates the sigma(E) pathway of stress response through a site- 2 cleavage of anti-sigma(E), RseA. Genes Dev 2002, 16:2147-2155.

49. Hayden JD, Ades SE: The extracytoplasmic stress factor, sigmaE, is required to maintain cell envelope integrity in Escherichia coli. PLoS One 2008, 3:e1573.

50. Papenfort K, Pfeiffer V, Mika F, Lucchini S, Hinton JC, Vogel J: SigmaEdependent small RNAs of Salmonella respond to membrane stress by accelerating global omp mRNA decay. Mol Microbiol 2006, 62:1674-1688,

51. Guisbert E, Rhodius VA, Ahuja N, Witkin E, Gross CA: Hfq modulates the sigmaE-mediated envelope stress response and the sigma32-mediated cytoplasmic stress response in Escherichia coli. J Bacteriol 2007, 189:1963-1973.

52. Swiercz JP, Hindra, Bobek J, Haiser HJ, Di Berardo C, Tjaden B, Elliot MA: Small non-coding RNAs in Streptomyces coelicolor. Nucleic Acids Res 2008, 36:7240-7251.

53. Saito S, Kakeshita H, Nakamura K: Novel small RNA-encoding genes in the intergenic regions of Bacillus subtilis. Gene 2009, 428:2-8.

54. Hsu LM, Zagorski J, Wang Z, Fournier MJ: Escherichia coli 6S RNA gene is part of a dual-function transcription unit. J Bacteriol 1985, 161:1162-1170.

55. Neusser T, Gildehaus N, Wurm R, Wagner R: Studies on the expression of 6S RNA from E. coli: involvement of regulators important for stress and growth adaptation. Biol Chem 2008, 389:285-297.

56. Neusser T, Polen T, Geissen R, Wagner R: Depletion of the non-coding regulatory $6 \mathrm{~S}$ RNA in E. coli causes a surprising reduction in the expression of the translation machinery. BMC Genomics 2010, 11:165.

57. Garza-Sanchez F, Gin JG, Hayes CS: Amino acid starvation and colicin D treatment induce A-site mRNA cleavage in Escherichia coli. J Mol Biol 2008, 378:505-519.

58. Steiner K, Malke H: relA-Independent amino acid starvation response network of Streptococcus pyogenes. J Bacteriol 2001, 183:7354-7364.

59. Paul BJ, Ross W, Gaal T, Gourse RL: rRNA transcription in Escherichia coli. Annu Rev Genet 2004, 38:749-770.

60. Eymann C, Homuth G, Scharf C, Hecker M: Bacillus subtilis functional genomics: global characterization of the stringent response by proteome and transcriptome analysis. J Bacterio/ 2002, 184:2500-2520.

61. Vera A, Aris A, Carrio M, Gonzalez-Montalban N, Villaverde A: Lon and ClpP proteases participate in the physiological disintegration of bacterial inclusion bodies. J Biotechnol 2005, 119:163-171.

62. Keiler KC: Physiology of tmRNA: what gets tagged and why?. Curr Opin Microbiol 2007, 10:169-175.

63. Weichart D, Querfurth N, Dreger M, Hengge-Aronis R: Global role for ClpPcontaining proteases in stationary-phase adaptation of Escherichia coli. J Bacteriol 2003, 185:115-125.

64. Elbein AD, Pan YT, Pastuszak I, Carroll D: New insights on trehalose: a multifunctional molecule. Glycobiology 2003, 13:17R-27R.

65. Flechard M, Fontenelle C, Blanco C, Goude R, Ermel G, Trautwetter A: RpoE2 of Sinorhizobium meliloti is necessary for trehalose synthesis and growth in hyperosmotic media. Microbiology 2010, 156:1708-1718.

66. Suarez R, Wong A, Ramirez M, Barraza A, Orozco Mdel C, Cevallos MA, Lara M, Hernandez G, Iturriaga G: Improvement of drought tolerance and grain yield in common bean by overexpressing trehalose-6-phosphate synthase in rhizobia. Mol Plant Microbe Interact 2008, 21:958-966.

67. Battesti A, Bouveret E: Acyl carrier protein/SpoT interaction, the switch linking SpoT-dependent stress response to fatty acid metabolism. Mol Microbiol 2006, 62:1048-1063.

68. Bott $\mathrm{M}$, Preisig $\mathrm{O}$, Hennecke $\mathrm{H}$ : Genes for a second terminal oxidase in Bradyrhizobium japonicum. Arch Microbiol 1992, 158:335-343.

69. Magnusson LU, Gummesson B, Joksimovic P, Farewell A, Nystrom T: Identical, independent, and opposing roles of ppGpp and DksA in Escherichia coli. J Bacteriol 2007, 189:5193-5202.

70. Chevance FF, Hughes KT: Coordinating assembly of a bacterial macromolecular machine. Nat Rev Microbiol 2008, 6:455-465.

71. Yang $X$, Ishiguro EE: Temperature-sensitive growth and decreased thermotolerance associated with relA mutations in Escherichia coli. J Bacteriol 2003, 185:5765-5771.

72. Dong T, Kirchhof MG, Schellhorn HE: RpoS regulation of gene expression during exponential growth of Escherichia coli K12. Mol Genet Genomics 2008, 279:267-277. 
73. Dahl JL, Kraus CN, Boshoff HI, Doan B, Foley K, Avarbock D, Kaplan G, Mizrahi V, Rubin H, Barry CE: The role of RelMtb-mediated adaptation to stationary phase in long-term persistence of Mycobacterium tuberculosis in mice. Proc Natl Acad Sci USA 2003, 100:10026-10031.

74. Hoffmann A, Bukau B, Kramer G: Structure and function of the molecular chaperone Trigger Factor. Biochim Biophys Acta 2010, 1803:650-661.

75. Gourion B, Sulser S, Frunzke J, Francez-Charlot A, Stiefel P, Pessi G, Vorholt JA, Fischer HM: The PhyR-sigma(EcfG) signalling cascade is involved in stress response and symbiotic efficiency in Bradyrhizobium japonicum. Mol Microbiol 2009, 73:291-305.

76. Martinez-Salazar JM, Sandoval-Calderon M, Guo X, Castillo-Ramirez S, Reyes A, Loza MG, Rivera J, Alvarado-Affantranger X, Sanchez F, Gonzalez V, Davila G, Ramirez-Romero MA: The Rhizobium etli $\mathrm{RpoH} 1$ and $\mathrm{RpoH} 2$ sigma factors are involved in different stress responses. Microbiology 2009, 155:386-397.

77. Sauviac L, Philippe H, Phok K, Bruand C: An extracytoplasmic function sigma factor acts as a general stress response regulator in Sinorhizobium meliloti. J Bacteriol 2007, 189:4204-4216.

78. Flechard M, Fontenelle C, Trautwetter A, Ermel G, Blanco C: Sinorhizobium meliloti rpoE2 is necessary for $\mathrm{H}(2) \mathrm{O}(2)$ stress resistance during the stationary growth phase. FEMS Microbiol Lett 2009, 290:25-31.

79. Reeve WG, Tiwari RP, Wong CM, Dilworth MJ, Glenn AR: The transcriptional regulator gene phrR in Sinorhizobium meliloti WSM419 is regulated by low pH and other stresses. Microbiology 1998, 144:3335-3342.

80. Fischer C, Geourjon C, Bourson C, Deutscher J: Cloning and characterization of the Bacillus subtilis prkA gene encoding a novel serine protein kinase. Gene 1996, 168:55-60

81. Tagourti J, Landoulsi A, Richarme G: Cloning, expression, purification and characterization of the stress kinase YeaG from Escherichia coli. Protein Expr Purif 2008, 59:79-85.

82. Masters M, Blakely G, Coulson A, McLennan N, Yerko V, Acord J: Protein folding in Escherichia coli: the chaperonin GroE and its substrates. Res Microbiol 2009, 160:267-277.

83. Eichenberger $\mathrm{P}$, Jensen $\mathrm{ST}$, Conlon EM, van Ooij C, Silvaggi J, GonzalezPastor JE, Fujita M, Ben-Yehuda S, Stragier P, Liu JS, Losick R: The sigmaE regulon and the identification of additional sporulation genes in Bacillus subtilis. J Mol Biol 2003, 327:945-972.

84. Erickson KD, Detweiler CS: The Rcs phosphorelay system is specific to enteric pathogens/commensals and activates ydel, a gene important for persistent Salmonella infection of mice. Mol Microbiol 2006, 62:883-894.

85. Andres Leon E, Ezkurdia I, Garcia B, Valencia A, Juan D: EcID. A database for the inference of functional interactions in E. coli. Nucleic Acids Res 2009, 37:D629-635

86. Michiels J, Moris M, Dombrecht B, Verreth C, Vanderleyden J: Differential regulation of Rhizobium etli rpoN2 gene expression during symbiosis and free-living growth. J Bacteriol 1998, 180:3620-3628.

87. Michiels J, Vande Broek A, Vanderleyden J: Molecular cloning and nucleotide sequence of the Rhizobium phaseoli recA gene. Mol Gen Genet 1991, 228:486-490

88. D'Hooghe I, Michiels J, Vlassak K, Verreth C, Waelkens F, Vanderleyden J: Structural and functional analysis of the fixL $J$ genes of Rhizobium leguminosarum biovar phaseoli CNPAF512. Mol Gen Genet 1995, 249:117-126.

89. Wren JD, Conway T: Meta-analysis of published transcriptional and translational fold changes reveals a preference for low-fold inductions. OMICS 2006, 10:15-27.

90. Vandesompele J, De Preter K, Pattyn F, Poppe B, Van Roy N, De Paepe A, Speleman F: Accurate normalization of real-time quantitative RT-PCR data by geometric averaging of multiple internal control genes. Genome Biol 2002, 3:RESEARCH0034.

91. Braeken K, Daniels R, Vos K, Fauvart M, Bachaspatimayum D, Vanderleyden J, Michiels J: Genetic determinants of swarming in Rhizobium etli. Microb Ecol 2008, 55:54-64.

92. RhizoBase. [http://genome.kazusa.or.jp/rhizobase/].

doi:10.1186/gb-2011-12-2-r17

Cite this article as: Vercruysse et al.: Stress response regulators identified through genome-wide transcriptome analysis of the (p)ppGppdependent response in Rhizobium etli. Genome Biology 2011 12:R17.

\section{Submit your next manuscript to BioMed Central and take full advantage of:}

- Convenient online submission

- Thorough peer review

- No space constraints or color figure charges

- Immediate publication on acceptance

- Inclusion in PubMed, CAS, Scopus and Google Scholar

- Research which is freely available for redistribution

Submit your manuscript at www.biomedcentral.com/submit 